\title{
T-Cadherin Expression Delineates Specific Regions of the Developing Motor Axon-Hindlimb Projection Pathway
}

\author{
Barbara J. Fredette and Barbara Ranscht \\ La Jolla Cancer Research Foundation, La Jolla, California 92037
}

T-cadherin is a unique member of the cadherin family anchored to the membrane by a glycosyl phosphatidylinositol moiety (Ranscht and Dours-Zimmermann, 1991). T-cadherin's distribution in the developing motor axon pathway was mapped by immunocytochemistry in the chick lumbosacral region as spinal neurons project to and innervate hindlimb muscle. On growing motor axons, T-cadherin was expressed biphasically. Initially, uniform T-cadherin expression occurred on motor neurons as they projected between the spinal cord and the base of the hindlimb (stage 21-24), and then decreased as the axons sorted to form dorsal, ventral and muscle nerve trunks (stage 25-27). Later, as motor axons entered and formed terminal axon arbors and synapses in muscle (stages 28-36), expression reoccurred heterogeneously among motor neuron pools. Thus, T-cadherin may guide the growth and fasciculation of all motor neurons during early axon extension, but only affect particular populations during the later expression period. In the mesenchyme of the motor axon pathway, T-cadherin was restricted to regions avoided by growing axons: the posterior-half sclerotome before and during the projection of motor axons through the T-cadherin-negative anterior half, and the extrasynaptic surfaces of developing muscle. The temporal and spatial expression patterns of T-cadherin and neurite outgrowth-promoting $\mathrm{N}$-cadherin were complementary both in nerve and muscle tissues. Thus, in the posterior sclerotome and in maturing muscle, T-cadherin may act as a negative regulator that works in concert with neurite growthpromoting molecules to guide motor axons to their peripheral targets.

[Key words: cadherins, axonal pathfinding, avoidance cues, motor neuron, neuromuscular development, synaptogenesis]

The establishment of synaptic connections during embryonic neural development depends on selective pathfinding by growing axons. In chick, innervation of skeletal muscles by somatic

\footnotetext{
Received Mar. 21, 1994; revised June 10, 1994; accepted June 16, 1994

Appreciation is extended to Dr. Matt Schibler for obtaining the confocal microscopy images, to Dr. Virginia Lee for generously supplying anti-neurofilament antibody RMO270, and to Dr. Lynn Landmesser, Fabiana Kubke, and Dr. Marc Tessier-Lavigne for discussing the distribution of CGRP. This work was supported by the National Institute of Health (Research Grant HD 25938 to B.R. and Postdoctoral Fellowship NS 09222 to B.F.), the March of Dimes Birth Defects Foundation (Basic Research Grant 0773 to B.R.), and the McKnight Foundation (to B.R.).

Correspondence should be addressed to Barbara Ranscht, La Jolla Cancer Research Foundation, 10901 North Torrey Pines Road, La Jolla, CA 92037.
}

Copyright (C) 1994 Society for Neuroscience $0270-6474 / 94 / 147331-16 \$ 05.00 / 0$ motor neurons occurs with a precise topography that develops by axonal reorganization at several positions along the motor neuron trajectory (Landmesser, 1978a,b; Landmesser and Morris, 1978; Hollyday, 1980; Lance-Jones and Landmesser, 1981). First, motor axons are segmentally segregated into spinal nerves by restricted growth through the anterior-half sclerotomes. Second, axons sort in the plexus regions at the base of the hindlimb into dorsal and ventral nerve trunks. Third, axons belonging to individual motor neuron pools segregate from the major nerve trunk, forming discrete muscle nerves, which, finally, branch and synapse within specific muscle targets. Selective muscle innervation by motor neurons occurs even when the segmental positions of motor neurons in the spinal cord, or the position of their muscle targets in the hindlimb are experimentally altered (Lance-Jones and Landmesser, 1980a,b).

The guidance of axons to their targets is controlled by the interaction of receptors on growth cone surfaces, such as the immunoglobulin-like cell adhesion molecules L1 and NCAM, integrins, and other neuronal cell surface components, with molecules in their environment. Molecules that support neurite outgrowth in vitro, such as NCAM, fibronectin, laminin, collagen IV, and heparan sulfate proteoglycan (Grumet et al., 1982; Rogers et al., 1983; Rutishauser et al., 1983; Silver and Rutishauser, 1984; Manthorpe et al., 1985), are present in the motor axon pathway prior to and during axon growth in regions traversed and avoided by motor axons such as the anterior and postcrior sclerotomes, respectively (Hay, 1978; Newgreen and Thiery, 1980; Rogers et al., 1986; Tosney et al., 1986; Hall et al., 1987). Therefore, the distribution of these molecules insufficiently explains specific route selection. More likely, motor axon guidance is the result of the combined function of widely distributed molecules that promote axon extension and discretely localized negative cues that restrict growth cone advance into specific regions (Keynes and Cook, 1990). Several possible inhibitory factors have been identified in regions avoided by growing motor axons, and include PNA-binding proteins of 48 and $55 \mathrm{kDa}$ (Stern et al., 1986; Davies et al., 1990), tenascin (Stern et al., 1989; Faissner and Kruse, 1990; Martini and Schachner, 1991) and chondroitin sulfate proteoglycan (Snow et al., 1990; Tosney and Oakley, 1990; Oakley and Tosney, 1991).

$\mathrm{T}$-cadherin, a functional member of the cadherin cell adhesion family anchored to. the plasma membrane through a glycosyl phosphatidylinositol moiety (Ranscht and Dours-Zimmermann, 1991; Vestal and Ranscht, 1992), is distributed in the posterior-half sclerotome during neural crest migration and early motor axon growth across the anterior sclerotome (Ranscht and Bronner-Fraser, 1991). In the present study, we have mapped the distribution of $\mathrm{T}$-cadherin during the projection of motor 
axons into the chicken hindlimb. This analysis revealed T-cadherin in regions avoided by extending motor axons and excluded from neuromuscular junctions. Thus, our observations are consistent with the possibility that $\mathrm{T}$-cadherin acts as a negative regulator of motor axon growth.

\section{Materials and Methods}

Embryos. Fertilized White Leghorn chicken cggs (McIntyre Poultry Farm, Lakeside, CA) were incubated in a force-draft incubator until the desired developmental stages. Stages were determined according to the criteria of Hamburger and Hamilton (1951).

Immunofluorescence. Embryos were dissected in phosphate-buffered saline (PBS, pH 7.2), and fixed for 2-6 hr in 4\% formaldehyde in PBS and then rinsed in several changes of PBS. Either the entire embryo, or for older stages (over st 30), the axial and hindlimb regions were cryoprotected overnight in 30\% sucrose in PBS. The tissue was embedded in O.C.T. (Miles Scientific, Elkhart, IN), frozen, and cryosectioned at $15 \mu \mathrm{m}$. The sections were collected on chrom-alum-coated microscope slides as three adjacent sets, each to be stained by indirect immunofluorescence with either anti-T-cadherin, anti-N-cadherin, or anti-neurofilament antibodies. $\mathrm{T}$-cadherin was visualized with a rabbit polyclonal antibody (1:150, Ranscht and Dours-Zimmermann, 1991), followed by fluorescein-conjugated goat anti-rabbit immunoglobulin (Ig) (1:150; Cappel, West Chester, PA). N-cadherin was detected with the monoclonal antibody GC4 (Volk and Geiger, 1984; purchased from Sigma, St. Louis). Nerve trunks were identified with monoclonal anti160 kDa neurofilament antibody RMO270 (Lee et al., 1987). Both GC4 and RMO270 were visualized with fluorescein-conjugated goat antimouse IgG (1:200; Antibodies, Inc., Davis, CA). All antibodies were diluted in PBS containing $2 \%$ bovine serum albumin, and PBS was used for rinsing. For intracellular staining, $0.1 \%$ Triton $\mathrm{X}-100$ was included with the antibody staining solution. As a control, $2 \%$ bovine serum albumin was used in place of the primary antibodies, and no immunoreactivity was observed. Staining was visualized and photographed by epifluorescence with a Zeiss Axiovert $405 \mathrm{M}$ inverted microscope. Photographs depicting comparisons of staining intensities were photographed and developed with identical exposure times.

Neuromuscular junctions were identified with fluorescein-conjugated $\alpha$-bungaroloxin ( $1 \mu \mathrm{g} / \mathrm{ml}$, Molecular Probes, Eugene, OR). For double labeling, the toxin was applied simultaneously with anti-T-cadherin, and anti-T-cadherin was detected with Texas red-conjugated donkey anti-rabbit lg (1:70, Amersham, Arlington Heights, IL). Staining was analyzed by laser scanning confocal microscopy using a Zeiss laser scanning microscope (LSM 410) equipped with argon $488 \mathrm{~nm}$ and helium/ neon $543 \mathrm{~nm}$ laser lines. Digital images of three optical sections, each approximately $1.5 \mu \mathrm{m}$ in thickness, were added, pseudocolored in either red (T-cadherin staining) or green ( $\alpha$-bungarotoxin), and contrast enhanced. The distribution of the two fluorochromes was determined by comparing the T-cadherin staining with the superimposed $\alpha$-bungarotoxin/T-cadherin image from the same field.

Electron microscopy. Five st 21-23 chick embryos were eviscerated and fixed at room temperature for $2 \mathrm{hr}$ in $4 \%$ paraformaldehyde and $0.2 \%$ glutaraldehyde in $0.12 \mathrm{~m}$ phosphate buffer, $\mathrm{pH} 7.3$, rinsed in 0.13 $\mathrm{M}$ phosphate buffer, $\mathrm{pH} 7.3$, and embedded in a mixture of gelatin (3\%) and chicken egg albumin (30\%). The resulting blocks were fixed with $4 \%$ paraformaldehyde in $0.12 \mathrm{M}$ phosphate buffer, and vibratome sectioned transverse to the spinal cord. Sections of 50-100 $\mu \mathrm{m}$ were collected in Tris-buffered saline (TBS, $0.1 \mathrm{~m}$ Tris, $77 \mathrm{~mm} \mathrm{NaCl}, \mathrm{pH} 7.6$ ), and incubated sequentially in the following reagents: $5 \%$ goat serum (1 $\mathrm{hr}$, room temperature), anti-T-cadherin antiserum $\left(1: 500,48 \mathrm{hr}, 4^{\circ} \mathrm{C}\right)$, 1:50 goat anti-rabbit Ig ( $1 \mathrm{hr}$, room temperature; Cappel) and 1:100 rabbit peroxidase-antiperoxidase $(1 \mathrm{hr}$, room temperature; Sternberger Monoclonals, Baltimore, MD). All of the immunoreagents were diluted in TBS containing $1 \%$ goat serum, and the sections were rinsed after each incubation step in three changes of TBS. As a final step, the sections were reacted in a chromogen mixture of $0.05 \%$ diaminobenzidine and $0.005 \%$ hydrogen peroxide. As a control, anti-T-cadherin antiserum was substituted with mouse RMO270, and reacted with anti-rabbit Ig. No DAB reaction product was observed when the inappropriate primary antibody was used. After immunostaining, the sections were postfixed with $1 \%$ osmium tetroxide, stained en bloc with $1 \%$ uranyl acetate, dehydrated, flat embedded in a TAAB/Epon (1:1) resin mixture, and polymerized for $2 \mathrm{~d}\left(65^{\circ} \mathrm{C}\right)$. The desired regions were cut out of the flatembedded sections, reembedded on larger plastic blocks, thin sectioned on an ultramicrotome, and collected on mesh grids. Some grids were stained with $0.2 \%$ lead citrate, and sections on the stained and unstained grids were viewed and photographed with a Hitachi $600 \mathrm{E}$ transmission electron microscope.

Di-I labeling of motor neurons. Motor neuron cell bodies were backlabeled from their muscle targets with the lipophilic red fluorescent dye Dil (1, 1'-dioctadecyl-3,3,3',3'-tetramethylindocarbocyanine perchlorate, Molecular Probes) (Honig and Hume, 1986). The spinal cord was exposed by ventral laminectomies in six st 31 embryos, and DiI $(0.3 \%$ in $100 \%$ ethanol) was injected into either the adductors or sartorius muscles on each side of the embryos. The injected embryos were incubated for $4 \mathrm{hr}$ in an oxygenated Tyrode buffer bath at $32^{\circ} \mathrm{C}$, fixed for $2 \mathrm{hr}$ with $4 \%$ paraformaldehyde, and processed for cryosectioning as described above. Serial sections transverse to the spinal cord were collected through the lumbosacral region, and the locations of Dil-labeled motor neurons in the lateral motor column were mapped by epifluorescence microscopy. Representative sections were photographed. The sections were subsequently processed for $\mathrm{T}$-cadherin immunofluorescence with a fluorescein (green) fluorophore, and DiI-labeled motor neurons were evaluated for the presence or absence of T-cadherin staining. Sections through the thigh were examined to verify that DiI was restricted to the injected muscles.

For cell culture experiments, motor neurons of six stage 27 embryos were backlabeled with DiI as described above. After the incubation period, the ventral portions of the lumbosacral spinal cords were isolated, and the cells dissociated by incubation for $30 \mathrm{~min}\left(37^{\circ} \mathrm{C}\right)$ with $0.05 \%$ trypsin in calcium-free Tyrode buffer. Glass round coverslips were coated with $0.2 \mathrm{mg} / \mathrm{ml}$ poly-D-lysine (about $40 \mathrm{kDa}$; Sigma) overnight, and were subsequently immersed in DMEM/F12 culture media (GIBCO-Bethesda Research Labs, Grand Island, NY) containing N2 additives (Bottenstein and Sato, 1979) and 10\% fetal calf serum (Gemini Bioproducts, Calabasas, CA). The neurons were plated over the coverslips at a density of $1.0 \times 10^{5}$ neurons/coverslip, and cultured for $15-$ $18 \mathrm{hr}$ at $37^{\circ} \mathrm{C}$ in a humidified $5 \% \mathrm{CO}_{2}$ incubator. The coverslips with attached neurons were fixed for 30 min with $4 \%$ formaldehyde in PBS, and immunofluorescently stained for T-cadherin as described above for the cryosections. The DiI-labeled motor neurons were evaluated for the presence or absence of T-cadherin immunofluorescence on their surfaces.

\section{Results}

The distribution of T-cadherin in the developing motor neuron pathway of the chick hindlimb was studied by indirect immunofluorescence with rabbit anti-T-cadherin antiserum (Ranscht and Dours-Zimmermann, 1991). Motor neuron axons project segmentally from the spinal cord across the anterior sclerotome during st 18-22 (Keynes and Stern, 1984), and converge to form, respectively, the crural and sciatic plexii at anterior and posterior regions at the base of the limb bud at about st 24 (LanceJones and Landmesser, 1981). There, the axons refasciculate into dorsal and ventral nerve trunks, and more distally beginning at st 26, into trunks projecting to individual muscles (LanceJones and Landmesser, 1981; Tosney and Landmesser, 1985a). The sequence of motor neuron growth out of the spinal cord and into the hindlimb was monitored by immunostaining of sections adjacent to those stained for T-cadherin with anti-neurofilament antibody (Lee et al., 1987).

\section{$T$-cadherin is biphasically expressed on motor neurons}

Early patterns. T-cadherin was first observed at a very low level and with a diffuse distribution in the lumbosacral spinal cord at st 18 (not shown), but appeared in the posterior regions of the epithelial somites at st 17 as previously described (see Fig. $10 A$ and Ranscht and Bronner-Fraser, 1991). At st 22 (Fig. $1 A, B^{\prime}$ ), as motor neuron genesis has ceased (Hollyday and Hamburger, 1977) and motor axons have projected out the spinal cord and into the anterior-half sclerotomes, intense $\mathrm{T}$-cadherin staining was present in the ventral spinal cord region, including the location of motor neuron cell bodies and the floor plate. In 

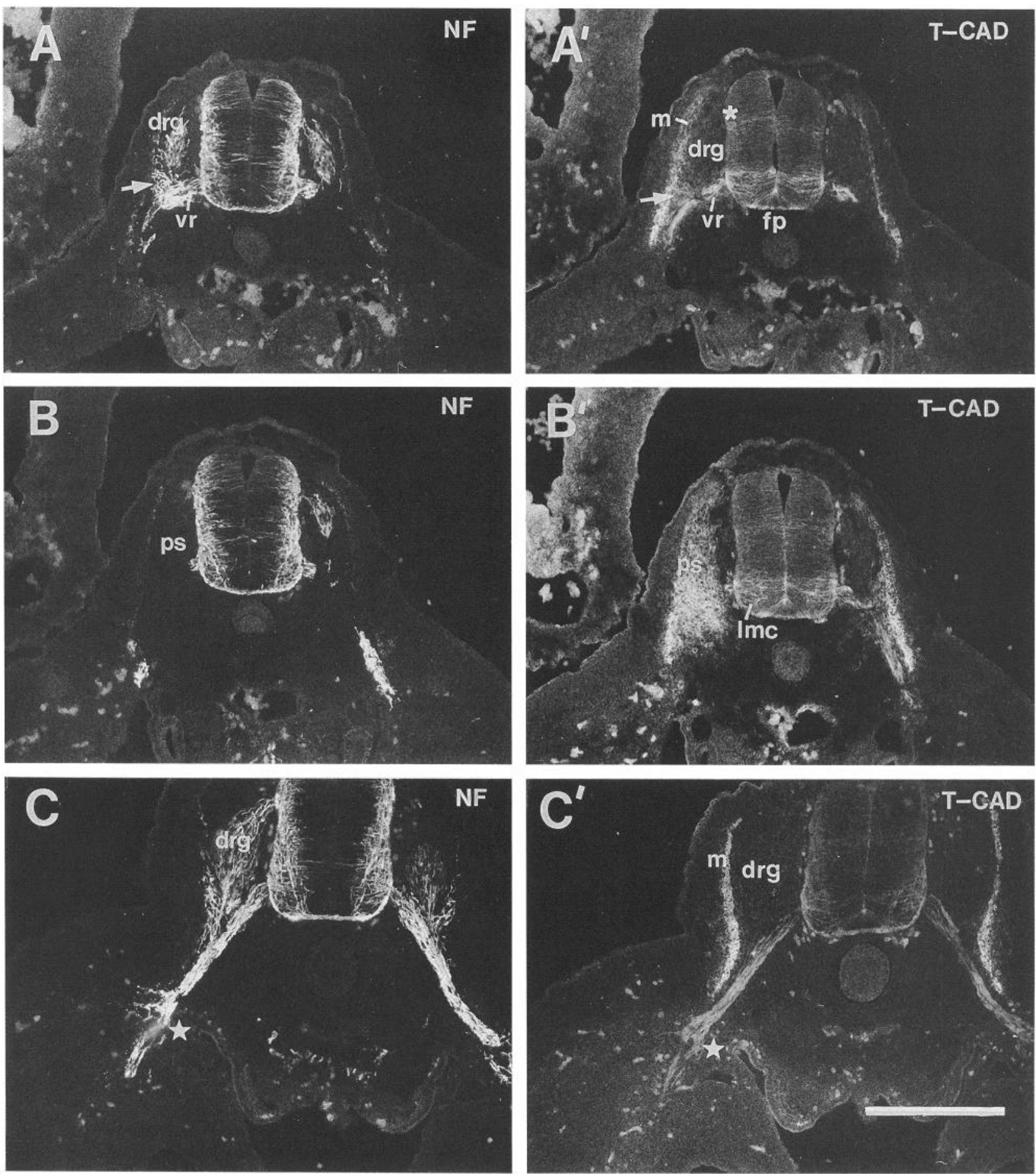

Figure 1. Distribution of T-cadherin in the lumbosacral region during early motor axon growth. $A-C$, Neurofilament immunofluorescence with RMO270 antibody. $A^{\prime}-C^{\prime}$, T-cadherin immunofluorescence in corresponding adjacent sections. $A$ and $A^{\prime}$, Sections cut transverse to the spinal cord through the anterior sclerotome region of a st 21 embryo as motor axons project out of the spinal cord as ventral roots (vr). Bright T-cadherin immunostaining is present in the ventral spinal cord, ventral roots, and myotomes. The remainder of the spinal cord (with the exception of a faintly labeled strip, indicated by the asterisk, corresponding to the location of forming commissural neurons), the surrounding mesenchyme and dorsal root ganglia $(d r g)$ are T-cadherin negative. Arrow points to faintly stained axons projecting towards the myotome $(m)$. $B$ and $B^{\prime}$, Sections from the same st 21 embryo. The left side of the section is cut through the posterior-half sclerotome ( $p s)$, which is brightly T-cadherin positive $\left(B^{\prime}\right)$ and avoided by motor axons $(B)\left(l m c\right.$, lateral motor column). $C$ and $C^{\prime}$, At st 24 T-cadherin immunostaining decreases in the spinal cord and in the spinal nerves projecting to the base of the limb and into the sciatic plexus (star), but remains bright in the myotome. Scale bar, $300 \mu \mathrm{m}$. 

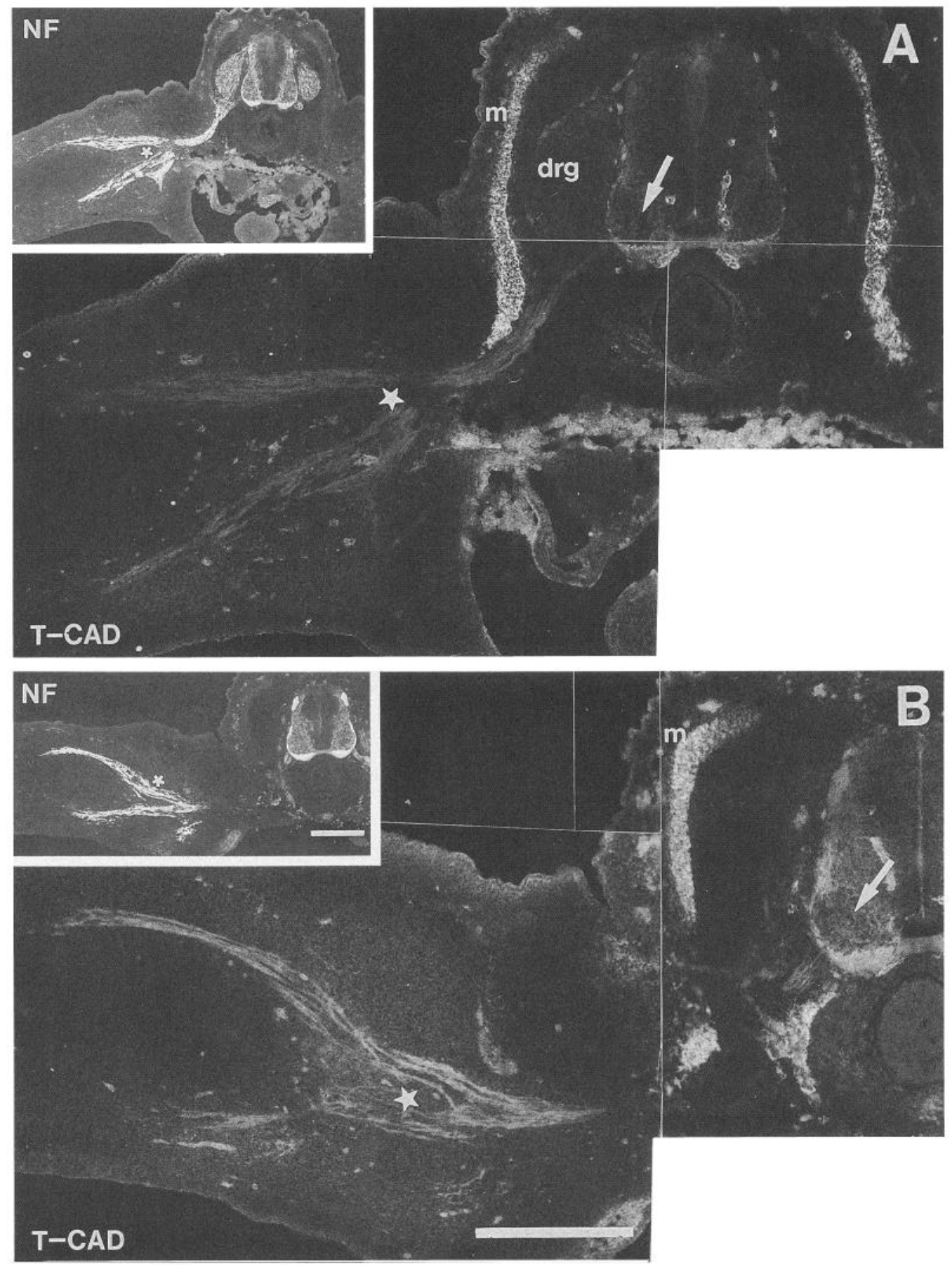

Figure 2. T-cadherin expression as motor axons project into the limb bud and innervate their muscle targets: T-cadherin immunofluorescence in sections cut transverse to the spinal cord and longitudinal to the limb. A. At st 26, as spinal nerves project far into the limb bud and sort into discrete nerve trunks, T-cadherin expression is barely visible in the lateral motor columns (arrow) and in spinal nerves, but is present on the blood vessels. Faint staining is detectable in the ventral funiculi of the spinal cord. The plexus region is indicated by a star. $B$, At st 28 , after motor axons have entered the muscle masses and have begun to form synapses, T-cadherin is reexpressed in the lateral motor columns (arrow) and spinal nerves. $\mathrm{T}$-cadherin also increases in the ventral and lateral funiculi. Insets, Neurofilament immunofluorescence in sections adjacent to $A$ and $B$. $m$, myotome; $d r g$, dorsal root ganglion. Scale bars, $300 \mu \mathrm{m}$.

addition, T-cadherin marked a cell population in the dorsal third of the spinal cord in a band-like pattern. T-cadherin was distributed in the ventral roots extending through the T-cadherinnegative anterior sclerotome (Fig. $1 A, A^{\prime}$ ), while immunoreactivity persisted in the posterior portion of the sclerotome (with respect to anterior-posterior or craniocaudal axis) (Fig. $1 B^{\prime}$ ), and was present in the myotomes. Thus, T-cadherin immu- nostaining was present in the lateral motor columns and spinal nerves containing motor neuron cell bodies and axons (respectively), and in regions avoided by growing motor axons (e.g., the floor plate and the posterior sclerotome) at early stages of motor neuron extension.

Innervation of hindlimb and epaxial muscles. As motor axons grew towards the base of the limb bud and converged to form 


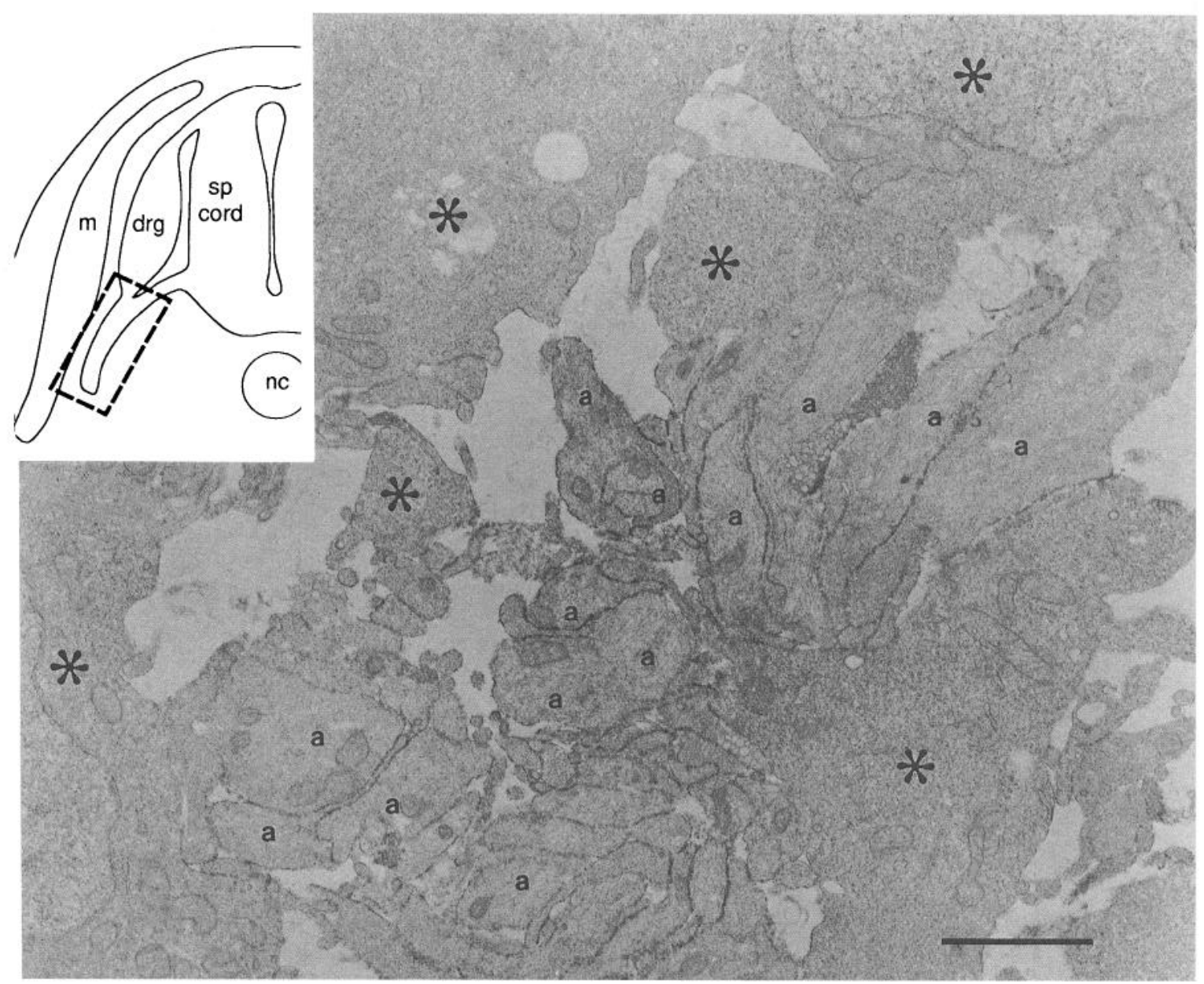

Figure 3. Ultrastructural localization of T-cadherin on spinal nerves at st 21: electron micrograph of spinal nerves that contain motor axons, peripherally projecting processes of dorsal root ganglia neurons and associated migratory cells, some of which are presumably Schwann cell precursors. T-cadherin is largely restricted to the surfaces of axonal profiles $(a)$, which are identified by the presence of intermediate filaments and microtubules, and the absence of polyribosomes. The associated non-neuronal cell bodies and processes (asterisks), distinguished from axons by their abundant polyribosomes, lack T-cadherin on their surfaces, except where they abut axonal profiles. Inset, Diagram to show the region (enclosed by dashed lines) analyzed by electron microscopy. $m$, myotome; $d r g$, dorsal root ganglion; $s p$ cord, spinal cord; $n c$, notocord. Scale bar, $2 \mu \mathrm{m}$.

the crural and sciatic plexii, T-cadherin immunostaining on motor axons remained high (Fig. $1 C, C^{\prime}$ ), but then began to decrease by st 24 . By st 26 (Fig. $2 A$ ), T-cadherin immunostaining was absent over the entire extent of motor neurons including their cell bodies in the lateral motor columns and their dorsal or ventral distal nerve trunks. T-cadherin remained absent from all nerve trunks as they separated to form projections to individual limb muscles, and as they first invaded the muscle masses at st 27 (Tosney and Landmesser, 1985b). By st 28, after motor axons had projected into the muscle and had begun branching to form synapses on myotubes (Dahm and Landmesser, 1988, 1991) (Fig. 2B), T-cadherin was reexpressed on motor neurons. Immunoreactivity progressively increased through st 33 , and persisted through st 36 after which the hindlimb nerves became permanently T-cadherin negative (compare Figs. 4, 5 to 8 ). This biphasic expression pattern on motor neurons is consistent with the biphasic T-cadherin mRNA profile in developing brain and spinal cord (Sacristán et al., 1993).

Motor axons innervating the epaxial muscles also transiently expressed $\mathrm{T}$-cadherin, but with a time course different from the hindlimb nerves. At st 21, ventral roots branching away from the spinal nerve and innervating the myotomes exhibited mod- erate $\mathrm{T}$-cadherin staining compared to the nerve trunk innervating the hindlimb (arrow, Fig. $1 A, A^{\prime}$ ). As in the hindlimb nerves, T-cadherin expression remained low until after st 27. However, on the epaxial motor neurons peak staining occurred at st 28 and had vanished by st 35 , whereas in the limb muscle motor neuron T-cadherin expression peaked at st 32 and persisted until st 36 . Thus, although the onset of T-cadherin expression occurred at the same time for both groups of motor neurons, the peak and down regulation of expression occurred at a faster rate for epaxial motor neurons than for limb motor neurons, and this difference may reflect an earlier time course of epaxial muscle innervation and maturation.

Immunofluorescence did not allow us to determine if T-cadherin in the growing nerves was located on motor neuron surfaces, or, instead, on the presumptive Schwann cells that migrate with the nerve trunks. Therefore, immunoelectron microscopic analysis of T-cadherin expression was performed on st 22 spinal cords and ventral roots (Fig. 3). The T-cadherin immunoreaction product was observed on the surfaces of the ventral root axons (identified by the presence of intermediate filaments and microtubules, and absence of polyribosomes), but was absent from, or only sparsely labeled, the surfaces of large cells in close 

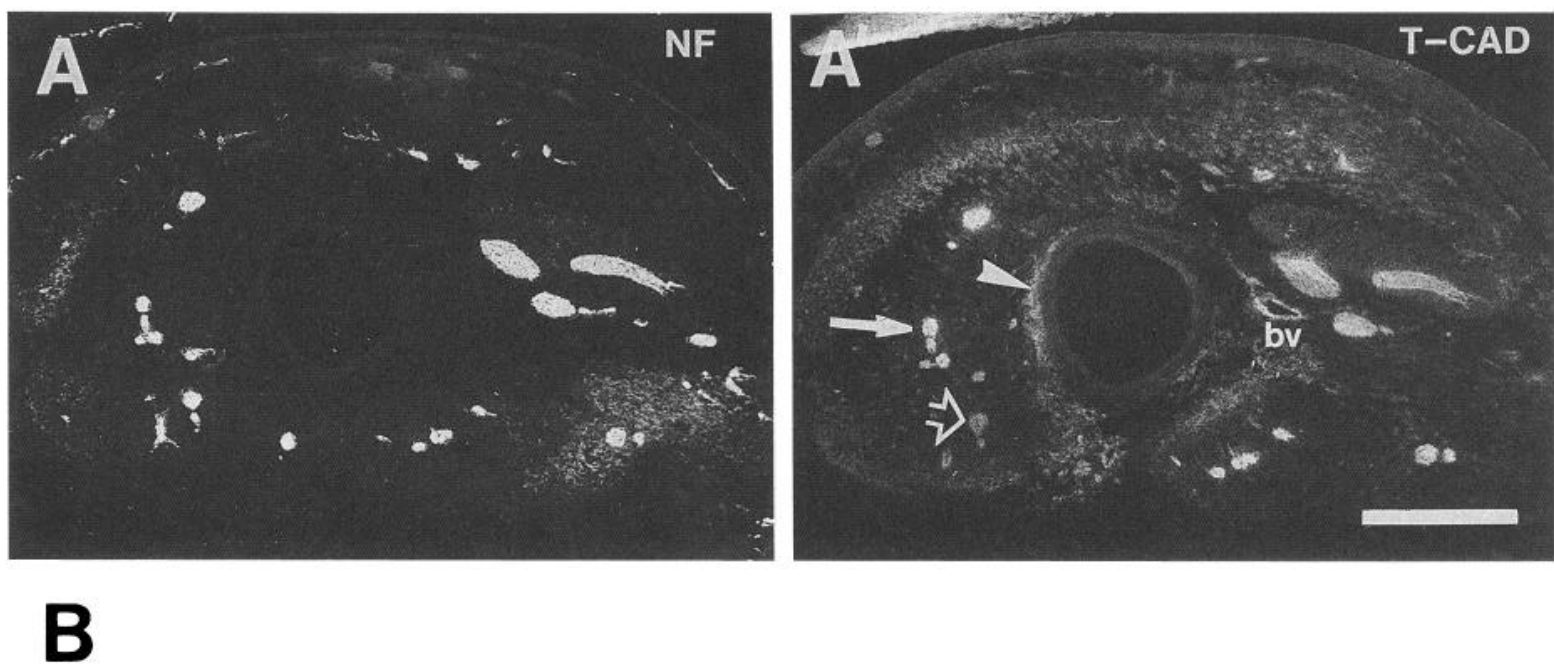

dorsal

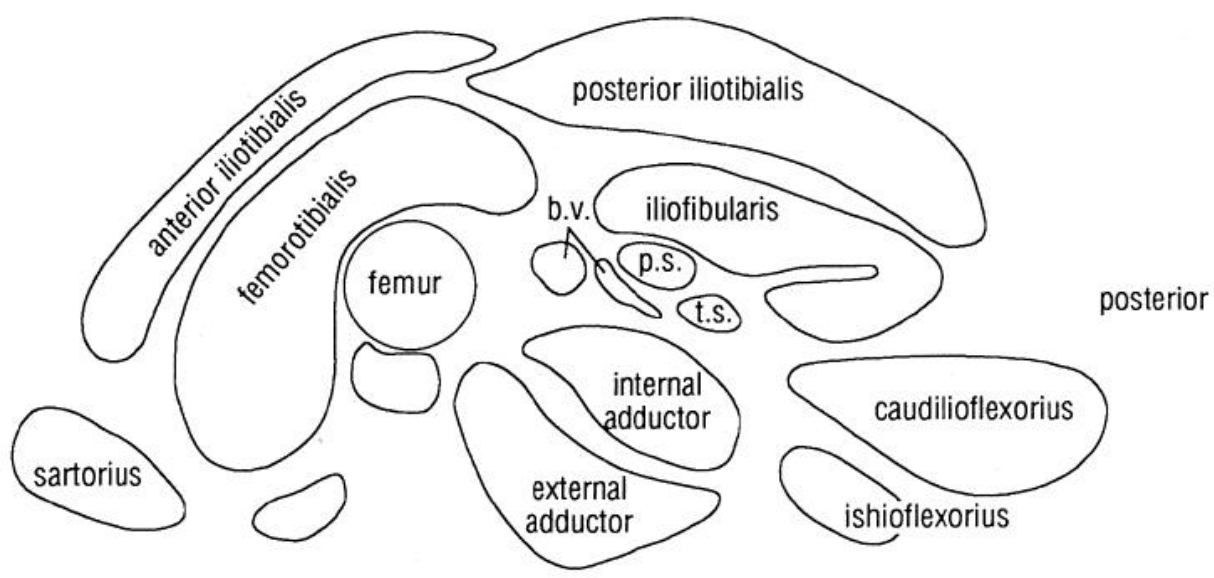

Figure 4. Nerve and muscle expression of T-cadherin at st 29 in the thigh, transverse plane. A, Neurofilament staining shows the positions of nerve trunks. $A^{\prime}$, T-cadherin immunofluorescence in an adjacent section. Nerve staining has increased as compared to st 28 , but is not uniform among the nerves. Examples of intense and low T-cadherin staining are indicated by solid and open arrows, respectively. T-cadherin staining is also visible on muscle cells, on blood vessels $(b v)$ and in the connective tissue near the femur (arrowhead). $B$, Diagram of thigh muscle positions. b.v., blood vessel; p.s., peroneal sciatic nerve; t.s., tibial sciatic nerve. Scale bar, $200 \mu \mathrm{m}$.

proximity to the axons, some of which are presumably migrating Schwann cell precursors. Thus, T-cadherin labeled motor axons. In the ventral spinal cord, cell bodies were T-cadherin-positive on their surfaces. Some of these cells could be identified as motor neurons by the projection of their attached axonal extensions out of the spinal cord (not shown). However, not all labeled cells could be identified as motor neurons, and therefore the possibility remains that additional populations of neuronal and/ or non-neuronal cells in the ventral spinal cord also express T-cadherin.

Heterogeneity of $T$-cadherin expression among motor neuron pools. T-cadherin immunoreactivity was homogeneously distributed among all portions of the lateral motor column and spinal nerves between st 18 and 24 , but after st 28 , staining varied among neuron populations belonging to discrete motor neuron pools. Figures 4 and 5 show the heterogeneous staining pattern among the intramuscular nerve trunks of the thigh muscles at st 29 and st 31 , respectively. The nerve trunks within the adductor muscles were intensely labeled for T-cadherin (Fig. $\left.5 A, A^{\prime}\right)$, but those in the sartorius muscle showed very low or no immunoreactivity (Fig. $5 B, B^{\prime}$ ). The caudilioflexorius nerves were also negative for T-cadherin, while nerve trunks in the posterior iliotibialis stained intermediately. The nerve trunks in the iliofibularis muscle exhibited a gradient of T-cadherin immunostaining intensity, according to their positions within the muscle (Fig. $5 C, C^{\prime}$ ). The positions of the nerve trunks in the anterior-posterior axis of the iliofibularis muscle are known to be topographically arranged with respect to the positions of the cell bodies in the motor neuron pool spanning from lumbosacral segment 3 to lumbosacral segment 7 of the spinal cord (Landmesser, 1978a,b). The T-cadherin-positive anterior nerve trunks in the muscle originate in the anterior portions of the spinal cord, while the posterior T-cadherin-negative nerve trunks originate in more posterior regions. These staining patterns were observed at all proximal to distal levels of the muscle nerves. Therefore, the observed differences were not due to the level of 

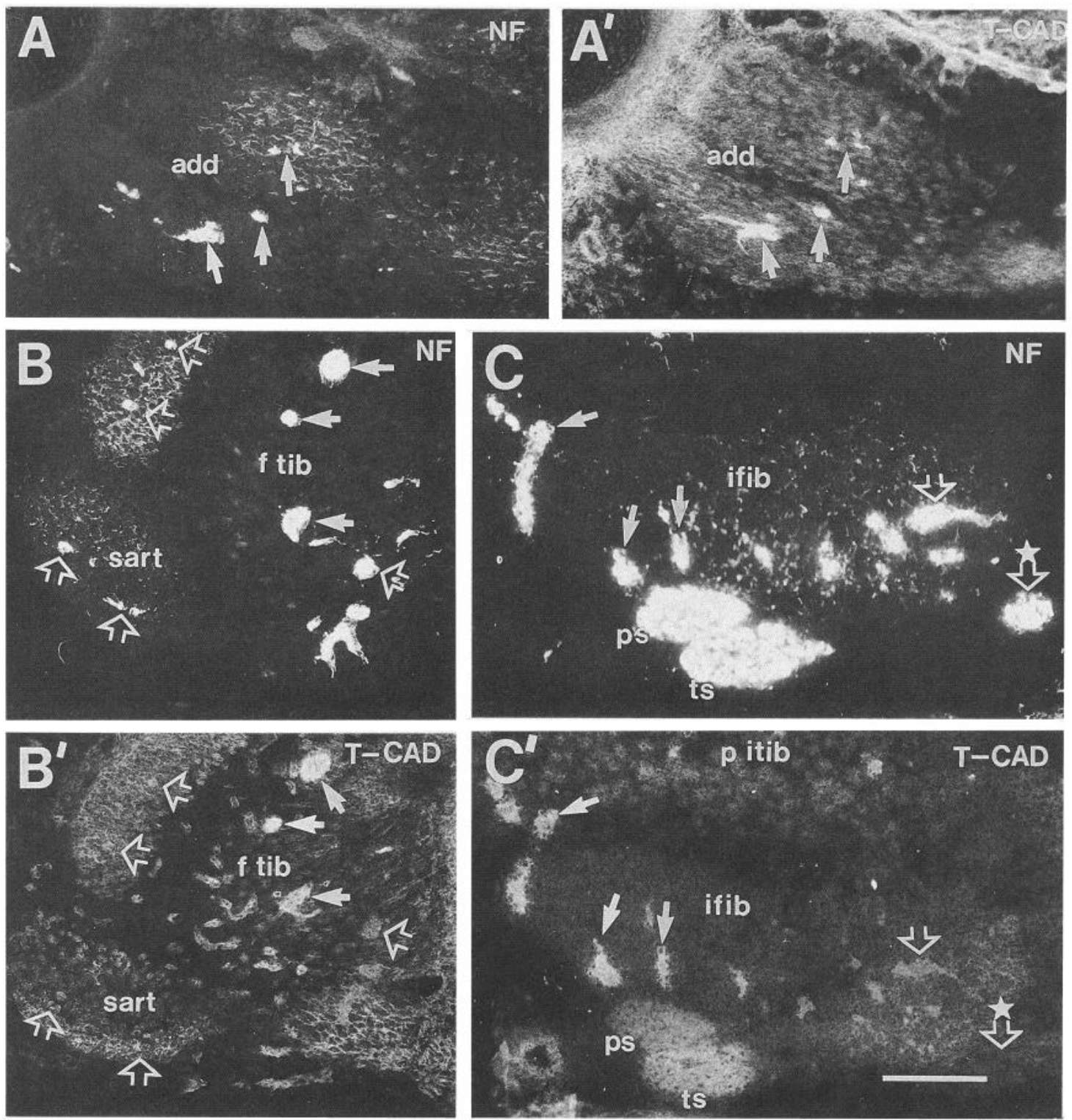

Figure 5. Heterogeneous T-cadherin immunofluorescence among nerve trunks of the thigh muscles at st 32 . $A-C$, Neurofilament immunofluorescence. $A^{\prime}-C^{\prime}$, Corresponding T-cadherin immunofluorescence. Solid arrows indicate intensely labeled nerves, and open arrows indicate negative or faintly labeled nerves. The nerve trunks indicated by solid arrows of the adductor $\left(a d d, A, A^{\prime}\right)$, femorotibialis $\left(f t i b, B, B^{\prime}\right)$, and anterior region of the iliofibularis muscles ( $i f i b, C, C^{\prime}$ ), are brightly T-cadherin positive, but nerve trunks indicated by open arrows in the sartorius muscle (sart, $\left.B, B^{\prime}\right)$ and the remainder of the femorotibialis and iliofibularis muscles exhibit low or negative T-cadherin staining. Also shown in $C^{\prime}$ is a portion of the posterior iliotibialis muscle ( $p$ itib) that contains T-cadherin-positive nerve trunks. Starred arrow in $C$ and $C^{\prime}$ points to the position of a T-cadherin-negative cutaneous nerve ventral to the iliofibularis muscle. Scale bar, $200 \mu \mathrm{m}$.

sectioning at particular regions of the nerves, which may occur if $\mathrm{T}$-cadherin levels varied with axonal distance from the spinal cord, or at branch points along the nerve.

In order to determine if the heterogeneity of $\mathrm{T}$-cadherin immunostaining in the lateral motor column is due to differences in expression levels by discrete motor neuron pools, motor neurons were backlabeled with $\mathrm{Di}-\mathrm{I}$ injected into individual thigh muscles at st 31 . Spinal cord sections containing the Di-I labeled neurons were immunostained for T-cadherin. The results, shown in Figure 6, revealed that the relative intensity differences observed in the intramuscular nerve trunks matched those among the motor neuron pools. Thus, the Di-I labeled cell bodies of $\mathrm{T}$-cadherin-negative sartorius nerves were located in the T-cadherin-negative region at the lateral aspect motor column in LS
1 and LS 2 (Fig. $6 A, A^{\prime}$ ), while the cell bodies of the T-cadherinpositive adductor nerves were located more medially in a region positive for T-cadherin (Fig. $6 B, B^{\prime}$ ). The heterogenous staining pattern of the thigh nerve trunks was retained between st 28 and 34 (Figs. 4, 5, 8A, $A^{\prime}$ ), after which T-cadherin expression began to decrease in all the nerves, persisting longest in the most intensely stained nerves, such as the adductor nerve. By st 36 , very low intramuscular nerve staining was visible in the hindlimb (Fig. $8 B, B^{\prime}$ ), and by hatching all the nerves innervating the muscle were $\mathrm{T}$-cadherin negative (not shown), although the perineurium ensheathing the extramuscular nerve trunks remained T-cadherin positive.

The heterogeneity of T-cadherin expression on motor neurons was confirmed by growing dissociated motor neurons at low 

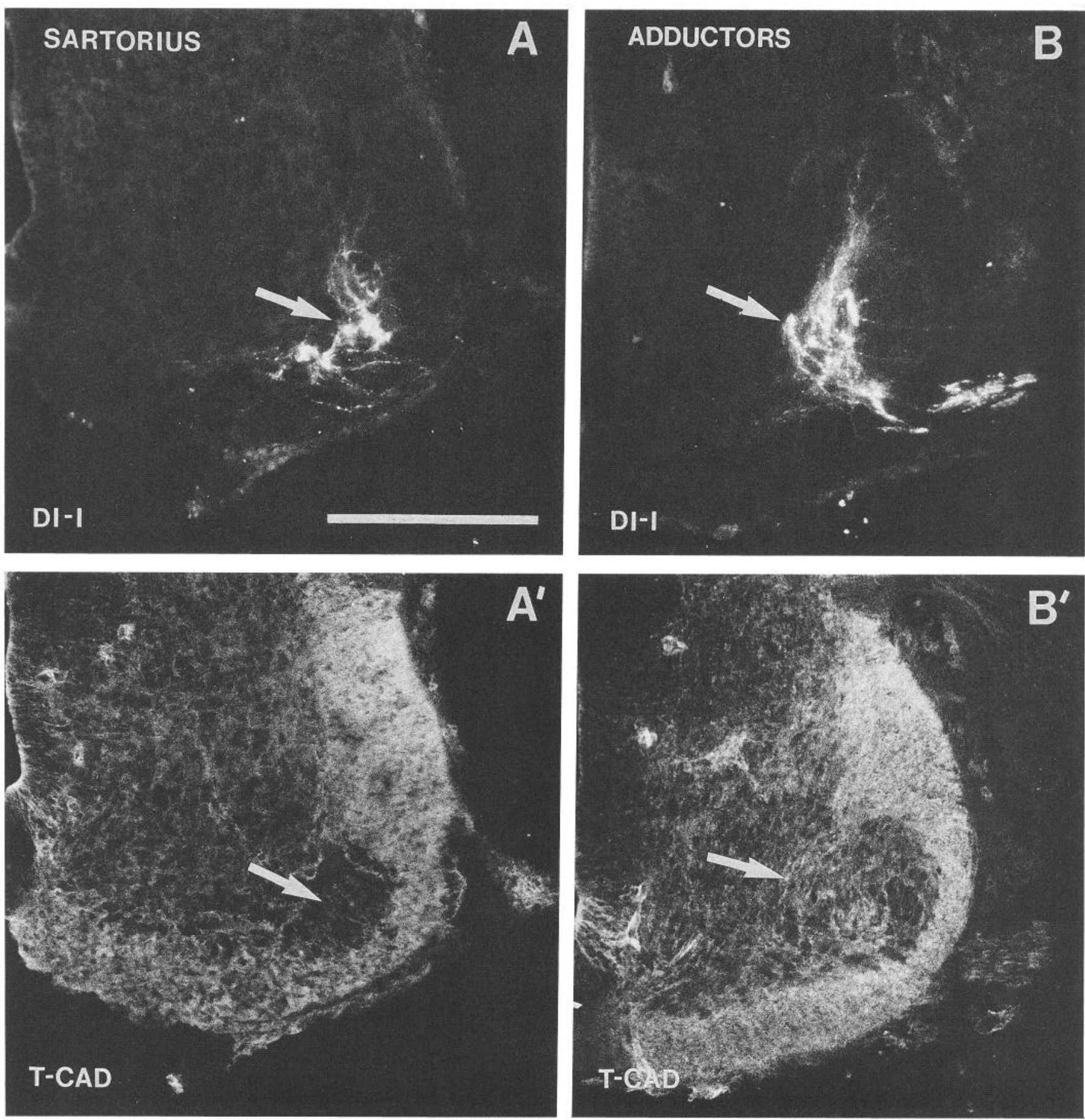

Figure 6. Heterogeneous T-cadherin immunostaining among motor neuron pools in the lateral motor column of the spinal cord corresponds with the pattern observed peripherally in the intramuscular nerve trunks. $A$ and $B$, Retrograde labeling of motor neuron pools by DiI injected into discrete thigh muscles. $A^{\prime}$ and $B^{\prime}, \mathrm{T}$-cadherin immunofluorescence in the same sections. Arrows point to the positions of DiI labeled motor neurons. The sartorius motor neurons in LS 1 are located in a T-cadherin-negative region of the lateral motor column $\left(A, A^{\prime}\right)$, but the adductor motor neurons in LS2 $\left(B, B^{\prime}\right)$ are located in T-cadherin-positive regions of the lateral motor column. Scale bar, $200 \mu \mathrm{m}$.

density in culture and immunofluorescently staining for the presence of T-cadherin. In order to identify motor neurons, DiI was injected in situ into the lumbosacral spinal nerves containing motor axons to back-label the motoneuron cell bodies in the spinal cord. The injections were made at stage 27 when $\mathrm{T}$-cadherin reexpression begins to occur heterogeneously. The ventral portions of the spinal cords were isolated, thus excluding any labeled sensory neurons, and the cells were dissociated and grown in culture. DiI persists in the motor neuron cell bodies, and is incorporated into the membranes of any new neurites extended in culture (Honig and Hume, 1986). In two separate experiments, many DiI labeled, brightly $\mathrm{T}$-cadherin-positive motor neurons were observed (Fig. 7). However, other motor neurons expressed varying levels of T-cadherin, and some motor 
neurons were T-cadherin negative. In one experiment, $60 \%$ of the motor neurons were brightly $\mathrm{T}$-cadherin positive, $36 \%$ were less intensely stained, and $4 \%$ were $\mathrm{T}$-cadherin negative; in the second experiment, these values were $25 \%, 30 \%$, and $45 \%$, respectively. (The different values between these two experiments are probably due to back-labeling of different proportions of high and low $\mathrm{T}$-cadherin-expressing motor neuron pools, since not all axons within the injected spinal nerves became labeled.) These experiments therefore demonstrate that motor neurons express T-cadherin, and that the expression after stage 27 is heterogeneous among motoneurons.

Taken together, these results show that $\mathrm{T}$-cadherin is expressed on the surfaces of motor axons in a biphasic temporal pattern: first, T-cadherin is homogeneously distributed on motor neurons projecting from the spinal cord into the plexus regions, and second, T-cadherin is heterogeneously expressed among motor neuron pools as motor axons innervate their muscle targets. Between these two phases, as motor axons exit the plexus region and grow to their muscle targets, T-cadherin expression is downregulated.

\section{T-cadherin in developing hindlimb muscles}

On muscle surfaces in the hindlimb, T-cadherin was first observed at st 29 , after motor neurons had invaded the muscles and had begun to form synapses on the myotubes (Fig. $4 A^{\prime}$ ). $\mathrm{T}$-cadherin immunoreactivity was first detected in the dorsally located anterior and posterior iliotibialis muscles, and also in the posterior region of the iliofibularis muscle. Slightly later (by st 32) (Fig. $8 A^{\prime}$ ), staining was also observed in the other thigh muscles, and progressively became more intense and uniform as development proceeded through hatching. At the latest time point observed, $4 \mathrm{~d}$ after hatching, T-cadherin immunoreactivity was strong in the limb muscle, and was abundant not only on myotube surfaces, but also in the extracellular spaces between myotubes. In addition to muscle, T-cadherin was detected on the newly formed connective tissue comprising the fascia between muscles, particularly at the posterior region of the thigh and at the junction between the thigh and the shank, and the perimysium ensheathing the muscles.

Staining postsynaptic regions of muscle cells with fluoresceinconjugated $\alpha$-bungarotoxin in combination with T-cadherin revealed that $\mathrm{T}$-cadherin was restricted to the extrasynaptic regions of muscle cells. At st 46 (Fig. 9) and at 4 d posthatching (not shown) staining for $\alpha$-bungarotoxin (green) and T-cadherin (red) did not overlap. This complementary distribution was thus observed when all $\mathrm{ACh}$ receptor clusters (identified by $\alpha$-bungarotoxin binding) are known to be postsynaptically localized(Dahm and Landmesser, 1991). Therefore T-cadherin is excluded from the neuromuscular junctions. This differential distribution may be due to differences between the synaptic and extrasynaptic basal lamina or the postsynaptic and extrasynaptic myotube cell membrane.

\section{Complementary expression of $T$-cadherin and $N$-cadherin in developing motor axon pathways}

By whole tissue analysis of T-cadherin mRNA, T-cadherin was previously found to be expressed simultaneously with $\mathrm{N}$-cadherin in the developing spinal cord, the somites, and in muscle (Sacristán et al., 1993). However, when the distribution and time course of T-cadherin was analyzed at the cellular level in the present study, expression patterns of these two cadherins
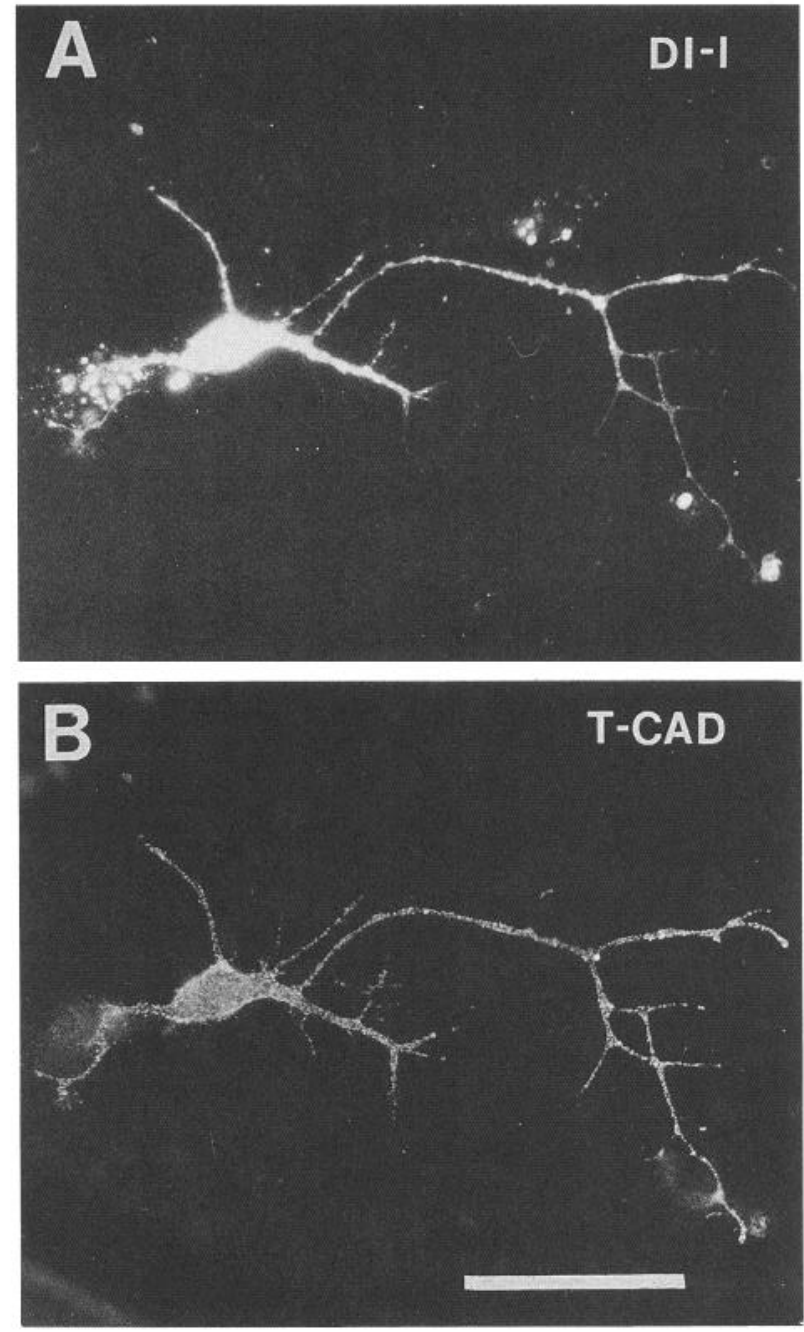

Figure 7. Motor neurons growing isolated in culture express T-cadherin. $A$, Motor neuron identified by Di-I back-labeling from its peripheral axons in situ. After $15 \mathrm{hr}$ in vitro, the Di-I persists in the soma and is incorporated into the newly formed neurites. $B$, T-cadherin immunofluorescence is present over the soma and processes of the same neuron. Scale bar, $55 \mu \mathrm{m}$.

were largely complementary. The expression of $\mathrm{N}$-cadherin has been previously described during spinal cord (Hatta and Takeichi, 1986; Hatta et al., 1987; Lagunowich et al., 1992; Redies et al., 1992) and hindlimb muscle development (Hatta et al., 1987; Hahn and Covault, 1992; Fredette et al., 1994), and thus we will restrict our description to a comparison with the expression of T-cadherin.

In the st 17 lumbosacral region, T-cadherin immunofluorescence was only detectable in the posterior portions of epithelial somites located anterior to the last three formed, but was not yet visible in the neural tube. In contrast, N-cadherin immunofluorescence was bright throughout the neural tube and surrounding somites (compare Fig. 10A, $A^{\prime}$ ). Since many developmental changes occur in an anterior to posterior gradient, temporal as well as spatial changes in the expression of T-cadherin and $\mathrm{N}$-cadherin were illustrated by comparing brachial and lumbosacral levels of st 20 embryos in horizontally cut sections (Fig. 10B, $B^{\prime}-D, D^{\prime}$ ). At the brachial level, the spinal cord, newly extended ventral roots, and the adjacent posterior 

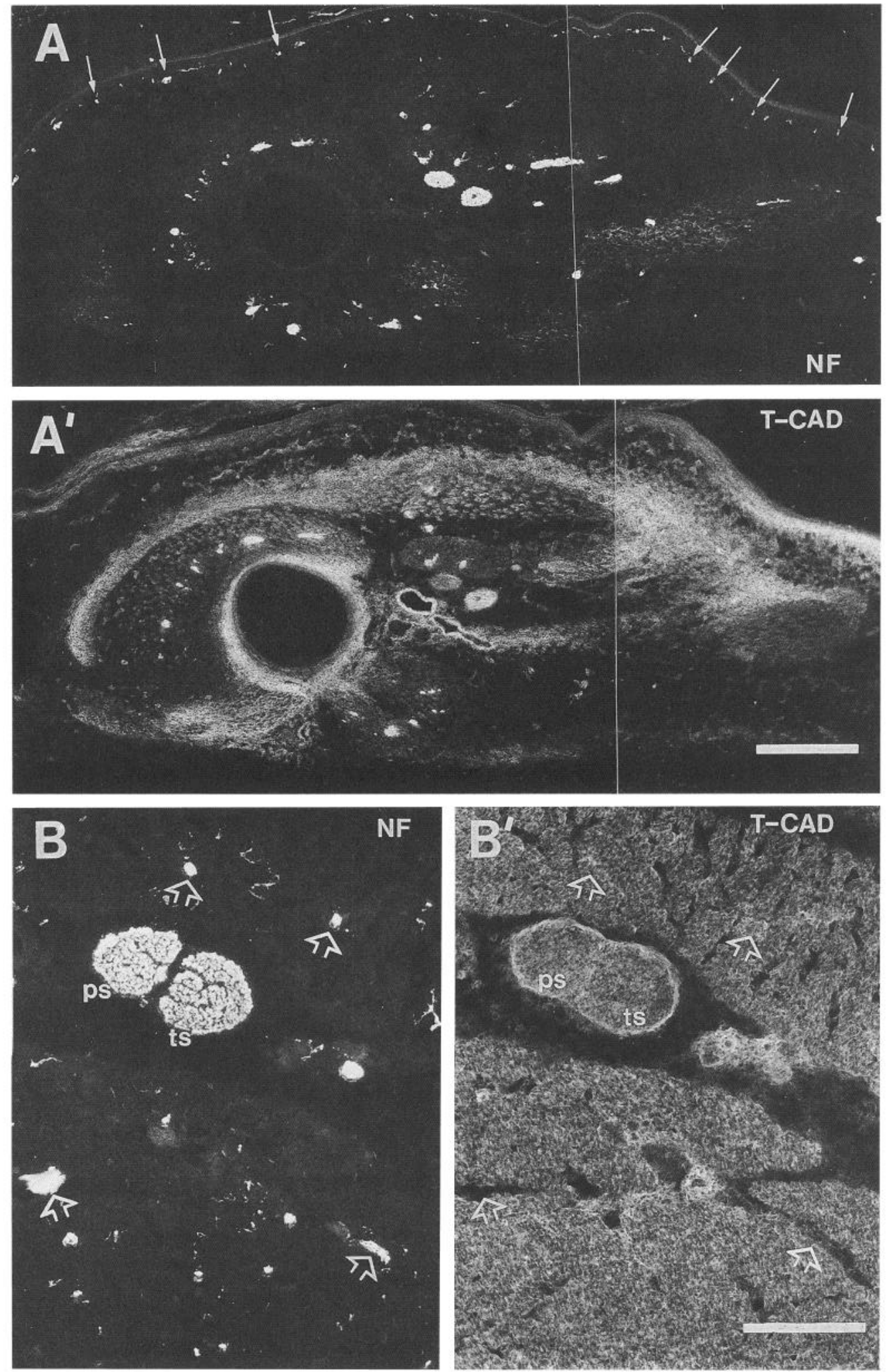

Figure 8. Changes in thigh nerve and muscle expression of T-cadherin between st 32 and 36 . For orientation, see diagram in Figure $4 B$. $A$ and $A^{\prime}$, Neurofilament $(A)$ and T-cadherin $\left(A^{\prime}\right)$ immunofluorescence in adjacent sections at st 32 . Nerve trunks and muscles are heterogeneously stained for T-cadherin, but the cutaneous sensory nerves (arrows) are T-cadherin negative (see Fig. $4 B$ for schematic of muscle names). Blood vessels and regions containing fibrous connective tissue are also T-cadherin positive. $B$ and $B^{\prime}$, Neurofilament $(B)$ and T-cadherin $\left(B^{\prime}\right)$ immunofluorescence of portions of the iliofibularis and adductor muscles, and sciatic nerve trunks in adjacent sections at st $361 / 2$. Muscle expression of T-cadherin has substantially increased compared to st 32 . However, the intramuscular nerves (open arrows) have become T-cadherin negative, and staining has decreased within the sciatic nerve trunks ps, peroneal sciatic; $t s$, tibial sciatic. Scale bars: $A$ and $A^{\prime}, 200 \mu \mathrm{m} ; B$ and $B^{\prime}, 500 \mu \mathrm{m}$. 

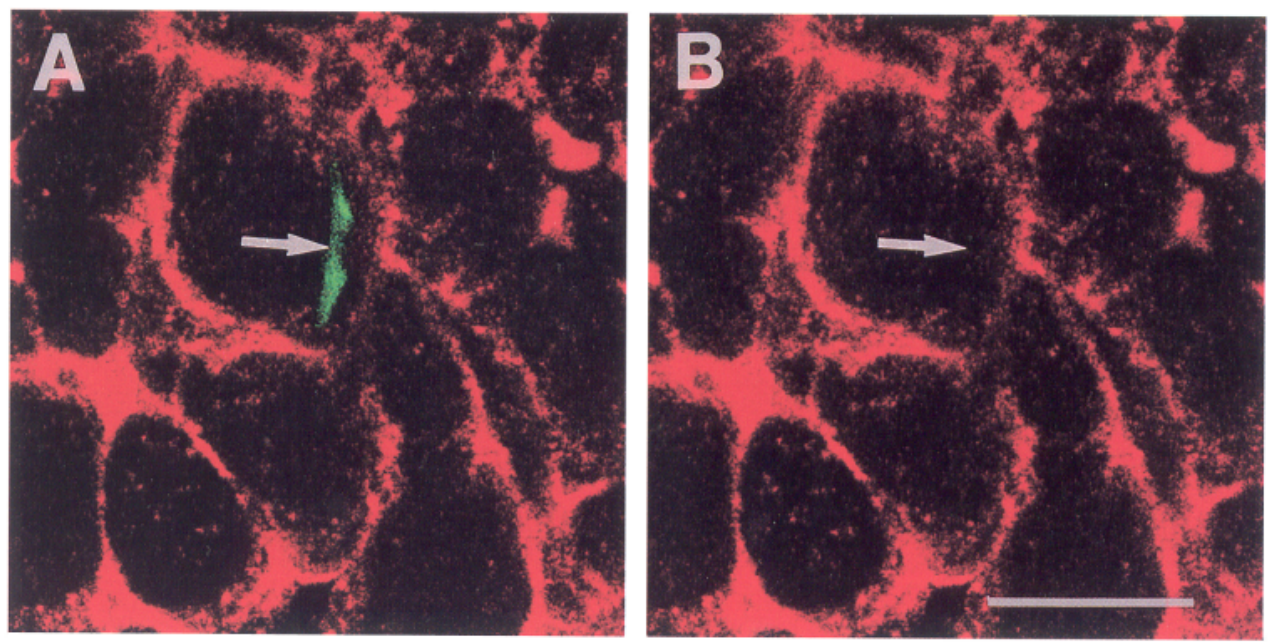

Figure 9. T-cadherin is restricted to the extrasynaptic regions in muscle: confocal image of st 46 (at hatching) posterior iliotibialis muscle immunostained for T-cadherin and, to identify the postsynaptic membrane surface, fluorescein-conjugated $\alpha$-bungarotoxin. $A$, Field with T-cadherin (red) and $\alpha$-bungarotoxin (green) images superimposed. $B$, The same field showing the T-cadherin image alone. Arrows indicate the position of an $\alpha$-bungarotoxin labeled synaptic region, which is negative for T-cadherin. Scale bar, $10 \mu \mathrm{m}$.

sclerotomes were brightly T-cadherin-positive (Fig. 10B), while in the lumbosacral region, $\mathrm{T}$-cadherin was less intense in these structures (Fig. 10C). In contrast, N-cadherin immunostaining was brighter at the lumbosacral level of the spinal cord than at the brachial level (Fig. $10 B^{\prime}, C^{\prime}$ ). Thus, the increase of T-cadherin in the spinal cord is complementary to a decrease of $\mathrm{N}$-cadherin. In the tissues surrounding the lumbosacral spinal cord, T-cadherin staining was present in the posterior-half sclerotomes, but $\mathrm{N}$-cadherin was restricted to the anterior-half sclerotomes where neural crest-derived cells were condensing to form the dorsal root ganglia (DRG) (Lallier and Bronner-Fraser, 1988) (Fig. 10D, $D^{\prime}$ ). Also, high levels of $\mathrm{N}$-cadherin, but low levels of $\mathrm{T}$-cadherin were apparent in this dorsal portion of the spinal cord. Both cadherins, however, were observed in the myotome.

After st 20, the distribution of both $\mathrm{N}$-cadherin and $\mathrm{T}$-cadherin continued to be largely complementary within the spinal cord (see Fig. $11 A, B$ ). Between st 20 and 31, N-cadherin immunostaining progressively increased and $\mathrm{T}$-cadherin immunostaining diminished in lateral portions of the dorsal funiculi, whereas the converse occurred in the medial portions of the dorsal funiculi and in the ventral funiculi. At st 31, the distribution of T-cadherin and $\mathrm{N}$-cadherin were complementary in the floor plate of the spinal cord, in the dorsal root ganglia (DRG) and sympathetic ganglia (Fig. 11 $A, B$ ). The sensory neurons in the DRG and their peripheral and central processes entering the dorsal horn were largely $\mathrm{N}$-cadherin positive. As an extension of the previous description by Redies et al. (1992) that a subpopulation of DRG neurons are $\mathrm{N}$-cadherin negative, we have observed that $\mathrm{T}$-cadherin is distributed on a minor population of DRG neurons (Fig. $11 C, D$ ). Correspondingly, the $\mathrm{N}$-cadherin-negative medial region of the dorsal funiculus was T-cadherin positive. Although we have not performed high magnification double fluorescence to determine if $\mathrm{T}$ - and $\mathrm{N}$-cadherin expression is mutually exclusive on individual cell bodies in the DRG, the segregation of staining in the dorsal funiculus would indicate that this is the case. Furthermore, sensory cutaneous nerve fibers in the limb are $\mathrm{N}$-cadherin positive but $\mathrm{T}$-cadherin negative, indicating that the $\mathrm{T}$-cadherin-positive sensory neurons in the DRG must belong to a sensory system other than the cutaneous afferents. The floor plate of the spinal cord and the sympathetic ganglia were positive for $\mathrm{N}$-cadherin, but negative for $\mathrm{T}$-cadherin (Fig. $11 C, D$ ). The con- nective tissue ensheathing the DRG and sympathetic ganglia was intensely labeled for $\mathrm{T}$-cadherin, but was negative for $\mathrm{N}$-cadherin.

An exception to the complementary expression of T-cadherin and N-cadherin was the simultaneous expression of both cadherins in myotomal muscle at st 20 . However, by st 31 , N-cadherin was downregulated in the myotome-derived axial muscles, while T-cadherin expression persisted (Fig. $10 C, D^{\prime}$, arrows). Similarly, the thigh muscles simultaneously expressed both cadherins between st 30 and 38 . However, $\mathrm{N}$-cadherin levels decrease in the hindlimb muscles after st 35 (Hahn and Covault, 1992) as $\mathrm{T}$-cadherin expression increased.

\section{Discussion}

\section{Biphasic expression of $T$-cadherin on motor neurons}

The present study revealed that $\mathrm{T}$-cadherin is expressed on motor axons and in the vicinity of their projection paths during two periods: (1) as they grow around the T-cadherin-positive posterior sclerotome, and (2) as they form terminal axonal arbors and synapses in their muscle targets. The two phases of $\mathrm{T}$-cadherin expression on growing motor axons correlate with particular events in the formation of the highly topographic motor axon-hindlimb muscle projection. Initially, T-cadherin is distributed uniformly on all motor axons as they exit the spinal cord and segregate into the segmental spinal nerves. Therefore, during this first phase of expression, T-cadherin may participate in recognition events that uniformly affect all motor axons, either by regulating axon fasciculation, or by acting as a receptor that renders all motor axons sensitive to specific guidance cues. During the second phase of expression, the heterogenous $\mathrm{T}$-cadherin distribution among motor neuron pools may render motor neurons differentially sensitive to contact with $\mathrm{T}$-cadherin ligands as these axons form terminal arbors and synapses within their muscle targets.

$\mathrm{T}$-cadherin levels in the limb muscle nerve trunks correlated with the levels observed in the locations of the corresponding motor neuron pools in the spinal cord. This argues that T-cadherin is expressed heterogeneously among motor neuron pools, and that the motor neurons, and not other cellular components either in the ventrolateral spinal cord (such as other neurons or glia), or in the muscle nerve trunks (such as sensory or sympathetic fibers), are responsible for the heterogeneity. Since the same pattern was observed at all proximal to distal levels of the 


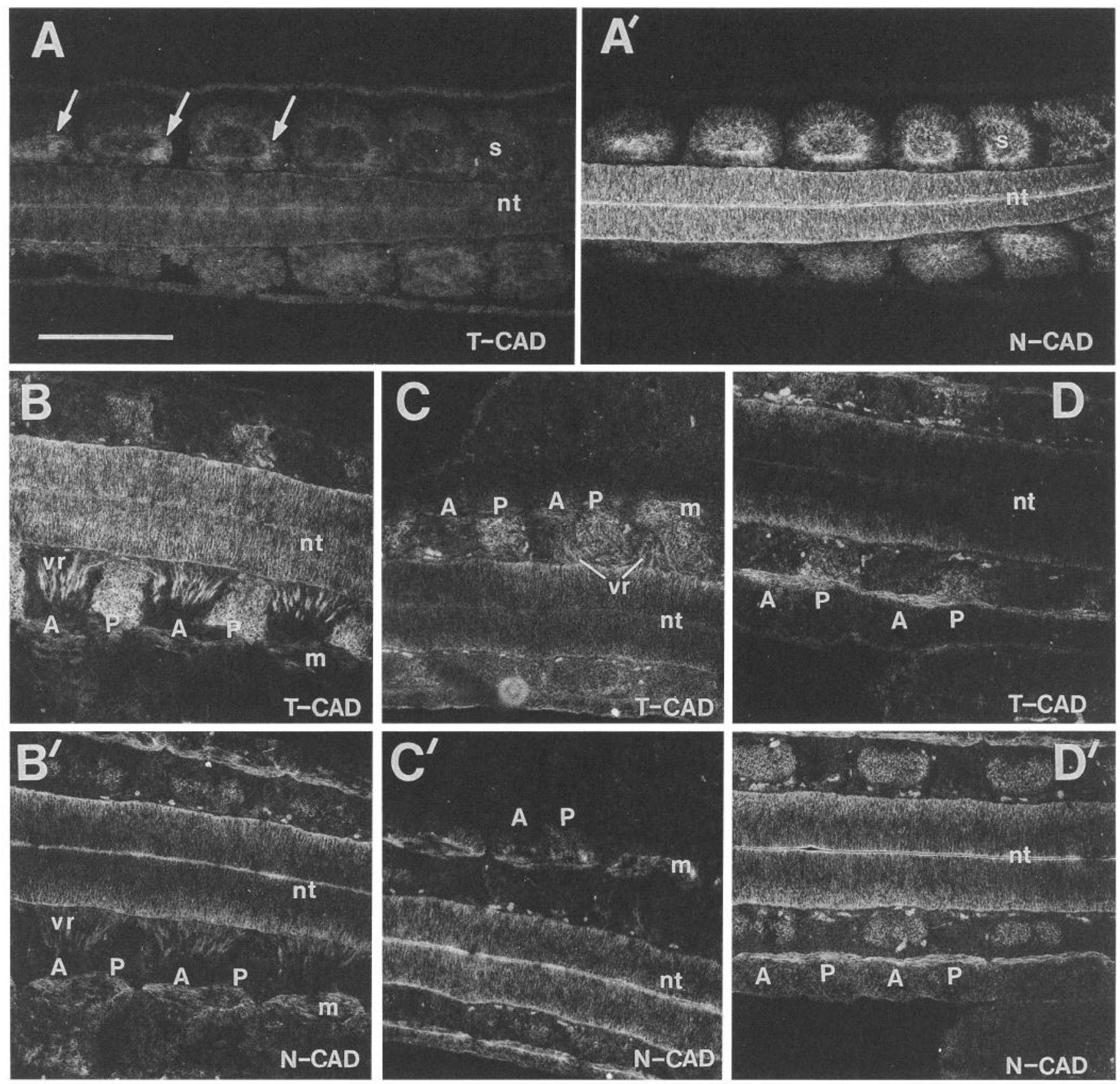

Figure 10. Complementary immunofluorescence patterns of T-cadherin and N-cadherin distribution, in horizontally cut sections, during early development. $A$ and $A^{\prime}$, T-cadherin $(A)$ and N-cadherin $\left(A^{\prime}\right)$ in st 17 neural tube $(n t)$ and adjacent somites $(s)$ of the lumbosacral region. The last formed somite is labeled $s$. T-cadherin is visible only in the posterior regions of the three somites indicated by arrows, but $\mathrm{N}$-cadherin is present throughout the neural tube and somites. $B$ and $B^{\prime}$ In the st 20 brachial region $(B)$, T-cadherin is abundant in the neural tube, emerging ventral roots $(v r)$, and posterior halves of the sclerotomes $(P)$, but is absent from the anterior halves $(A)$, traversed by the ventral roots. In an adjacent section $\left(B^{\prime}\right)$, N-cadherin staining is low in all of these structures. The myotomes $(m)$ express both N-cadherin and T-cadherin. $C$ and $C^{\prime}$, In the st 20 lumbosacral region, T-cadherin $(C)$ is much less abundant than in the brachial region, while the converse is true for $\mathrm{N}$-cadherin $\left(C^{\prime}\right)$. $D$ and $D^{\prime}$, In a section dorsal to the one shown in $C$ and $C^{\prime}$, T-cadherin $(D)$ is present in the posterior sclerotome, but $\mathrm{N}$-cadherin $\left(D^{\prime}\right)$ is detected in the anterior sclerotome where dorsal root ganglia are forming. Only the lateral region of the spinal cord and the myotomes express both $\mathrm{N}$-cadherin and T-cadherin. Scale bar, $300 \mu \mathrm{m}$.

muscle nerves between st 28 and 36, the variations are not due to the growth state of the nerves, such as distance from the spinal cord or position at branch points. Furthermore, we have observed by in situ hybridization heterogeneous expression of $\mathrm{T}$-cadherin mRNA in the lateral motor columns (unpublished observations), and by immunofluorescence that motor neurons grown dissociated in culture express varying levels of T-cadherin on their surface, although in neither of these cases were the motor neuron projection targets identified. Collectively, our data indicate that between st 28 and 34, T-cadherin is expressed heterogeneously among motor neuron pools.

The variation of T-cadherin levels among motor neuron populations after st 28 is one of few examples of molecular heterogeneity among motor neurons. Other cases are the calcitonin 

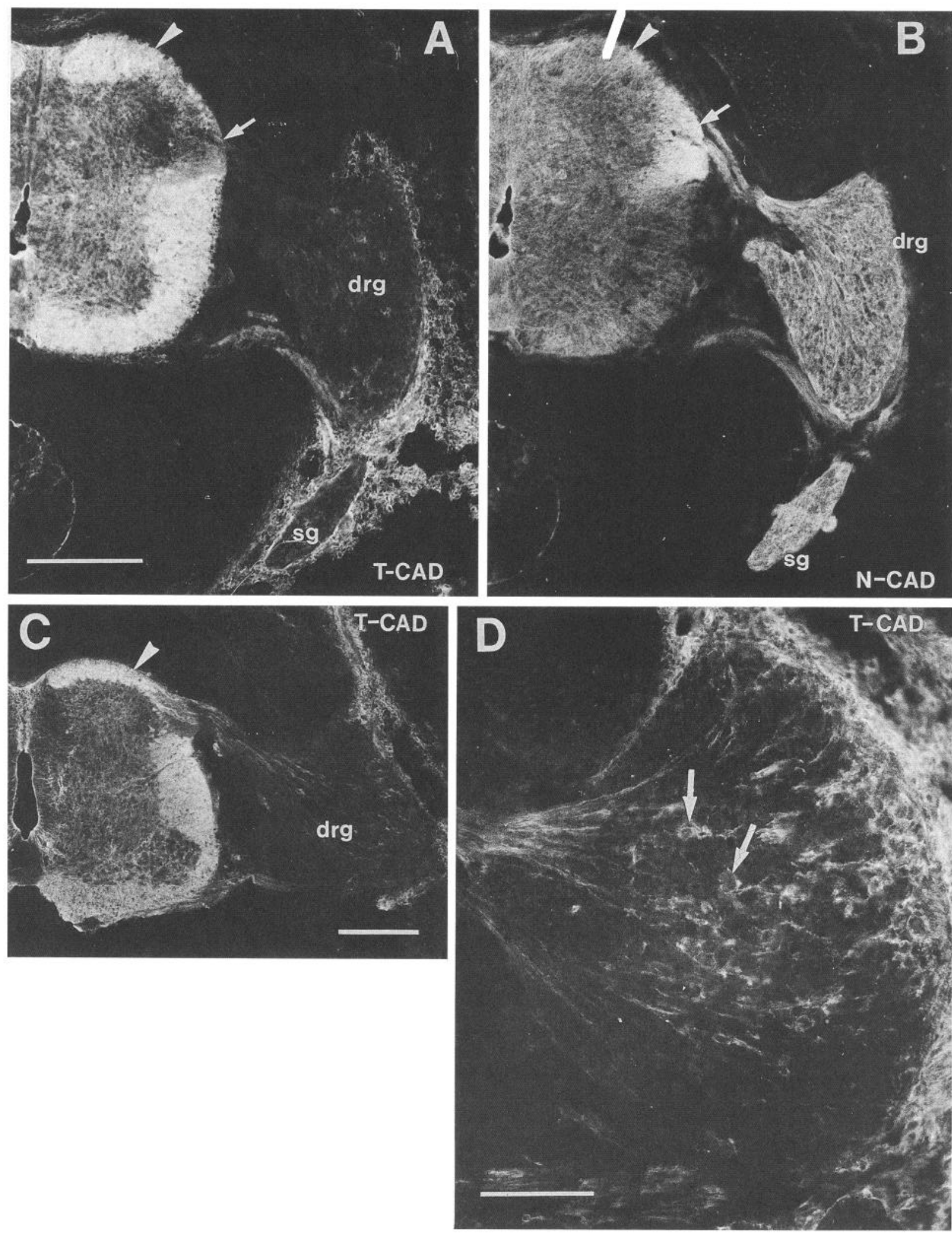

Figure 11. Complementary T-cadherin and N-cadherin expression in st 31 spinal cord and surrounding ganglia. $A$ and $B$, Double T-cadherin $(A)$ and $\mathrm{N}$-cadherin $(B)$ immunofluorescence of a thoracic spinal cord section. T-cadherin staining $(A)$ is intense in the ventral funiculus and the medial portion of the dorsal funiculus (arrowhead) and low in the lateral portion of the dorsal funiculus (arrow), while the converse is observed for $\mathrm{N}$-cadherin $(B)$. Complementary staining also occurs in the dorsal root ganglia $(d r g)$ and in the sympathetic ganglia (sg), which are bright for $\mathrm{N}$-cadherin, but low for T-cadherin. T-cadherin is strongly expressed in the connective tissue ensheathing the peripheral ganglia. $C$, In a low magnification view of a T-cadherin immunostained section, a population of T-cadherin-positive neurons can be seen projecting from the dorsal root ganglia and into the T-cadherin-positive medial region of the dorsal funiculus in the spinal cord (arrowhead, $D$, High-magnification view of T-cadherin-positive neurons in the dorsal root ganglia. Scale bars: $A-C, 200 \mu \mathrm{m} ; D, 100 \mu \mathrm{m}$. 
gene-related peptide (CGRP) (Kubke and Landmesser, unpublished observations; Tessier-Lavigne and Mudge, 1987) and polysialylated NCAM (Tang et al., 1992). The distribution of these three molecules overlaps, but does not exactly correspond. The expression of polysialylated NCAM in motor axon trunks correlates with their projection to ventral regions of the developing limb, and enzymatic removal of polysialic acid perturbs the ability of axons to project ventrally (Tang et al., 1992). T-cadherin, however, is transiently downregulated as motor axons sort into dorsal and ventral nerve trunks and into discrete muscle nerves that project between the base of the hindlimb and their muscle targets. Therefore, a function of axonal T-cadherin in dorsal-ventral sorting of nerve trunks can be excluded.

Like T-cadherin, CGRP varies among motor neuron pools and is apparent after st 28. However, CGRP is expressed simultaneously with T-cadherin on some motor neuron pools, while others express only one of the two molecules. For example, motor axons innervating the internal adductor and tibialis anterior muscles express both CGRP and T-cadherin, but the sartorius motor neurons are T-cadherin negative and CGRP positive and the opposite is true for the femorotibialis and posterior iliotibialis motor neurons (Tessier-Lavigne and Mudge, 1987). Furthermore, CGRP expression persists after T-cadherin expression on motor axons has ceased. The significance of this heterogeneity is not clear for either molecule, since the patterns do not correspond to any known functional (fast vs slow, extensor vs flexor) or positional patterns. Since the heterogeneous pattern of $\mathrm{T}$-cadherin appears during branching and synapse formation of motor axons in the muscle, the pattern most likely relates to one of these events.

\section{T-cadherin expression in muscle}

$\mathrm{T}$-cadherin expression in muscle occurs after the initial period of myotube formation and persists well after myogenesis is completed. Unlike NCAM, N-cadherin, and M-cadherin that are expressed in developing muscle and have been shown to be involved in myoblast fusion (Covault and Sanes, 1986; Knudsen et al., 1990a,b; Donalies et al., 1991; Hahn and Covault, 1992; Moore and Walsh, 1993), T-cadherin is unlikely to be involved in this process in vivo because it is expressed too late.

$\mathrm{T}$-cadherin expression in muscle correlates with the cessation of motor axon growth, and the formation of terminal axon arbors and synapses. $\mathrm{T}$-cadherin is excluded from neuromuscular junctions and is restricted to the extrasynaptic regions. This localization is complementary to that of NCAM (Covault and Sanes, 1986) and N-cadherin (Cifuentes-Diaz et al., 1994), which become restricted to the neuromuscular junction around hatching. The postsynaptic region is selectively reinnervated after denervation (Sanes et al., 1978), and the distribution of $\mathrm{T}$-cadherin is consistent with the putative role as a barrier to axonal sprouting. According to this hypothesis, T-cadherin would function in the arrest of motor neuron growth and synapse formation in a mode opposite to that proposed for S-laminin in the synaptic cleft (Hunter et al., 1989): T-cadherin may act to prevent axonal sprouting onto the extrasynaptic muscle surface, while S-laminin in the synaptic cleft may serve to "anchor" the axon at the synaptic site. However, it remains to be determined if T-cadherin is initially present at neuromuscular contacts and only later becomes excluded from maturing synaptic structures or if it is segregated to extrasynaptic surfaces from the onset of its expression.
Complementary expression of $T$-cadherin and $N$-cadherin in neural and mesenchyme tissues

A minor subpopulation of developing sensory neurons in the dorsal root ganglia contains $\mathrm{T}$-cadherin and we suggest that this population complements the $\mathrm{N}$ - and R-cadherin-positive neurons of the DRG. The centrally projecting processes of T-cadherin-positive sensory neurons project to a $\mathrm{T}$-cadherin-positive medial region of the dorsal funiculus that is $\mathrm{N}$ - and R-cadherin negative (this study and Redies et al., 1992). The T-cadherinpositive subpopulation is probably part of the long distance, rostrally projecting fiber system, whereas the N-cadherin-positive neurons enter the lateral region containing short distanceprojecting fibers (Redies et al., 1992). Furthermore, in our study, because cutaneous nerve trunks in the periphery express $\mathrm{N}$-cadherin, but not T-cadherin, this functional group of neurons can be classified as T-cadherin negative. Therefore, while heterogeneous expression of cell surface molecules has previously been described in sensory neurons (Dodd et al., 1984), heterogeneity in cadherin expression among particular functional groups of sensory neurons during axon sorting, pathfinding and synapse formation may be indicative of cadherins participating in the segregation of sensory axons into functional groups.

Our observation that $\mathrm{T}$-cadherin and $\mathrm{N}$-cadherin expression patterns are complementary in virtually all of the tissues examined suggests that their regulation is linked in some way. This complementarity was apparent also in regions where the two cadherins are colocalized, in that their expression levels change temporally in opposite ways, so that as one cadherin is upregulated, the other decreases. In these regions, for example, the neural tube and the myotomal and skeletal muscles, $\mathrm{N}$-cadherin expression occurs before T-cadherin expression, thus implying that individual cells are capablc of simultaneously expressing both cadherins. It seems, however, that the expression of both $\mathrm{T}$-cadherin and $\mathrm{N}$-cadherin is temporarily restricted and, in the long run, the expression of the two cadherins is mutually exclusive.

\section{T-cadherin as a negative regulator of motor axon growth?}

Avoidance mechanisms have been hypothesized to participate in molor axon pathfinding during their segmental projection through the somitic mesoderm (Keynes and Stern, 1984, 1988; Tosney, 1988; Davies et al., 1990), during growth through specific channels in the presumptive pelvis (Tosney and Landmesser, 1984; Oakley and Tosney, 1991), and finally as they cease growing and form synaptic contacts on their muscle targets (Hunter et al., 1989). The discrete distribution of T-cadherin on motor axons and in regions of their projection path that are avoided during axon growth is highly suggestive that $\mathrm{T}$-cadherin acts as an avoidance cue to restrict the pathway of motor axons. $\mathrm{T}$-cadherin is expressed segmentally in the posterior-half sclerotome before and during the growth of motor axons through the anterior sclerotome, and therefore, T-cadherin is a likely candidate for participating in the early motor axon organization. Motor axons extend from the spinal cord all along the anteriorposterior neuraxis, and are then funneled through the anterior sclerotome, avoiding the posterior half, to yicld the scgmental organization of spinal nerves. The molecular signals for the segmental growth of motor axons reside within the sclerotome, as motor axons project through the anterior sclerotome even when presented in the posterior position under experimental 
conditions (Keynes and Stern, 1984). Inhibitory molecules located in the posterior sclerotome may play an important role in the segmental organization of motor axons, and T-cadherin's restriction to this region is consistent with a role in this process. Other molecular candidates expressed in the posterior sclerotome and putatively responsible for the segmental growth of motor axons are the peanut agglutinin-binding proteins of 48 and $55 \mathrm{kDa}$, which have growth conc collapse activity (Stern et al., 1986; Davies et al., 1990) and chondroitin-6-sulfate (Oakley and Tosney, 1991; Snow et al., 1990). Tenascin is also restricted to the posterior sclerotome as motor neurons project through the anterior region, but experimental alteration of tenascin expression in this region does not disrupt the segmental organization of motor axons (Rickmann et al., 1985; Stern et al., 1989). The restricted distribution of permissive molecules, such as the $70 \mathrm{kDa}$ molecule M7412 (Tanaka et al., 1989) and butyrylcholinesterase (Layer et al., 1988) in the anterior halves of sclerotomes may also contribute to segmental motor axon growth by providing strongly attractive guidance cues.

Other areas avoided by growing motor axons are the perinotocordal region and the presumptive pelvis, where chondrocyte condensation and subsequent bone formation occurs. $\mathrm{T}$-cadherin is present in portions of the presumptive vertebrae derived from the posterior sclerotome, but is not expressed in the remainder of the perinotocord region or in the presumptive femur region, nor is it expressed on motor axons as they grow past these regions. Therefore, T-cadherin is unlikely to be involved in axon avoidance of these regions, while both PNAbinding proteins and chondroitin-6-sulfate (Oakley and Tosney, 1991) could contribute to growth cone avoidance of these particular areas in the motor axon pathway.

The present study has revealed a distinct and unique expression pattern of T-cadherin during the projection of motor axons into hindlimb muscles that predicts a role of $\mathrm{T}$-cadherin as a negative regulator of motor axon guidance. Initial experiments carried out in this laboratory indicate that $\mathrm{T}$-cadherin substrates significantly reduce neurite outgrowth in vitro (Fredette and Ranscht, 1993), and thus provide experimental support for this hypothesis. The formation of the motor axon pathway depends on the specific recognition of multiple extracellular guidance cues by neuronal growth cone receptors and the work presented here suggests that T-cadherin is likely to be an important molecular player contributing to the development of the stereotyped innervation pattern of the chick hindlimb muscle.

\section{References}

Bottenstein JE, Sato GH (1979) Growth of a rat neuroblastoma cell line in serum-free supplemented medium. Proc Natl Acad Sci USA 76:514-517.

Cifuentes-Diaz C, Nicolet M, Goudou D, Reiger F, Mege RM (1994) $\mathrm{N}$-cadherin expression in developing, adult and denervated chicken neuromuscular systemr: accumulation at both the neuromuscular junction and the node of Ranvier. Development 120:1-11.

Covault J, Sanes JR (1986) Distribution of NCAM in synaptic and extrasynaptic portions of developing and denervated skeletal muscle. J Cell Biol 102:731-739.

Dahm LM, Landmesser LT (1988) The regulation of intramuscular nerve branching during normal development and following activity blockade. Dev Biol 130:621-644.

Dahm LM, Landmesser LT (1991) The regulation of synaptogenesis during normal development and following activity blockade. J Neurosci $11: 238-255$.

Davies JA, Cook GM, Stern CD, Keynes RJ (1990) Isolation from chick somites of a glycoprotein fraction that causes collapse of dorsal root ganglion growth cones. Neuron 4:11-20.
Dodd J, Solter D, Jessell TM (1984) Monoclonal antibodies against carbohydrate differentiation antigens identify subsets of primary sensory neurons. Nature 311:469-472.

Donalies M, Craner M, Ringwald M, Starzinski-Powitz A (1991) Expression of M-cadherin, a member of the cadherin multigene family, correlates with differentiation of skeletal muscle cells. Proc Natl Acad Sci USA 88:8024-8028.

Faissner A, Kruse J (1990) J1/tenascin is a repulsive substrate for central nervous system neurons. Neuron 5:627-637.

Fredette B, Ranscht B (1993) Distribution and function of T-cadherin during the projection of motor axons to the hindlimb. Soc Neurosci Abstr 19:1481.

Fredette B, Rutishauser U, Landmesser L (1994) Regulation and activity-dependence of N-cadherin, NCAM isoforms, and polysialic acid on chick myotubes during development. J Cell Biol 123:18671888.

Grumet M, Rutishauser U, Edelman GM (1982) Neural cell adhesion molecule is on embryonic muscle cells and mediates adhesion to nerve cells in vitro. Nature 295:693-695.

Hahn C, Covault J (1992) Neural regulation of N-cadherin gene expression in developing and adult skeletal muscle. J Neurosci 12:46774687.

Hall DE, Neugebauer KM, Reichardt LF (1987) Embryonic neural retinal cell response to extracellular matrix proteins: developmental changes and effects of the cell substratum attachment antibody (CSAT). J Cell Biol 104:623-634.

Hamburger V, Hamilton HL (1951) A series of normal stages in the development of the chick embryo. J Morphol 88:49-82.

Hatta K, Takeichi M (1986) Expression of N-cadherin adhesion molecules associated with early morphogenic events in chick development. Nature 320:447-449.

Hatta K, Takagi S, Fujisawa H, Takeichi M (1987) Spatial and temporal expression pattern of $\mathrm{N}$-cadherin cell adhesion molecules correlated with morphogenetic processes of chicken embryos. Dev Biol 120:215-227.

Hay ED (1978) Fine structure of embryonic matrices and their relation to the cell surface in ruthenium red-fixed tissues. Growth $42: 399$ 423.

Hollyday M (1980) Organization of motor pools in the chick lumbar lateral motor column. J Comp Neurol 194:143-170.

Hollyday M, Hamburger V (1977) An autoradiographic study of the formation of the lateral motor column in the chick embryo. J Comp Neurol 132:197-208.

Honig MG, Hume RI (1986) Fluorescent carbanocyanine dyes allow living neurons of identified origin to be studied in long-term cultures. J Cell Biol 103:171-186.

Hunter DD, Shah V, Merlie JP, Sanes JR (1989) A laminin-like adhesive protein concentrated in the synaptic cleft of the neuromuscular junction. Nature 338:229-234.

Keynes R, Cook G (1990) Cell-cell repulsion: clues from the growth cone? Cell 62:609-610.

Keynes RJ, Stern CD (1984) Segmentation in the vertebrate nervous system. Nature 310:786-789.

Keynes RJ, Stern CD (1988) Mechanisms of vertebrate segmentation. Development 103:413-429.

Knudsen KA, McElwee SA, Myers L (1990a) A role for the neural cell adhesion molecule, NCAM, in myoblast interaction during myogenesis. Dev Biol 138:159-168.

Knudsen KA, Myers L, McElwee SA (1990b) A role for the $\mathrm{Ca}^{2+}$ dependent adhesion molecule, $\mathrm{N}$-cadherin, in myoblast interaction during myogenesis. Exp Cell Res 188:175-184.

Lagunowich LA, Schneider JC, Chasen S, Grunwald GB (1992) Immunohistochemical and biochemical analysis of $\mathrm{N}$-cadherin expression during CNS development. J Neurosci Res 32:202-208.

Lallier T, Bronner-Fraser M (1988) A spatial and temporal analysis of dorsal root and sympathetic ganglia formation in the avian embryo. Dev Biol 127:90-112.

Lance-Jones C, Landmesser L (1980a) Motoneuron projection patterns in embryonic chick limb following partial deletions of the spinal cord. J Physiol (Lond) 302:559-580.

Lance-Jones C, Landmesser L (1980b) Motoneuron projection patterns in the chick hindlimb following early partial reversals of the spinal cord. J Physiol (Lond) 302:581-602.

Lancc-Jones C, Landmcsser L (1981) Pathway selection by embryonic chick lumbosacral motor neurons during normal development. Proc R Soc Lond [Biol] 214:1-18. 
Landmesser L (1978a) The distribution of motor neurones supplying chick hind limb muscles. J Physiol (Lond) 284:371-389.

Landmesser $\mathrm{L}(1978 \mathrm{~b})$ The development of motor projection patterns in the chick limb. J Physiol (Lond) 284:391-414.

Landmesser L, Morris DG (1978) The development of functional innervation in the hind limb of the chick embryn. J Physiol (Iond) 249:301-326.

Layer PG, Alber R, Rathjen FG (1988) Sequential activation of butyrylcholinesterase in rostral half somites and acetylcholinesterase in motor neurones and myotomes preceding growth of motor axons. Development 102:387-396.

Lee V, Carden M, Schlaepfer W, Trajanowski JQ (1987) Monoclonal antibodies distinguish several differentially phosphorylated states of the two largest neurofilament subunits (NF-H and NF-M) and demonstrates their existence in the normal nervous system of rats. J Neurosci 7:3474-3488.

Manthorpe M, Davies GE, Varon S (1985) Purified proteins acting on cultured chick embryo ciliary ganglion neurons. Fed Proc 44:27532759.

Martini R, Schachner M (1991) Complex expression pattern of tenascin during innervation of the posterior limb buds of the developing chicken. J Neurosci Res 28:261-279.

Moore R, Walsh FS (1993) The cell adhesion molecule M-cadherin is specifically expressed in developing and regenerating, but not denervated skeletal muscle. Development 117:1409-1420.

Newgreen D, Thiery JP (1980) Fibronectin in early avian embryos: synthesis and distribution along the migratory pathways of neural crest cells. Cell Tissue Res 211:269-291.

Oakley RA, Tosney KW (1991) Peanut agglutinin and chondrin-6sulphate are molecular markers for tissues that act as barriers to axon advance in the avian embryo. Dev Biol 147:187-206.

Ranscht B, Bronner-Fraser M (1991) T-cadherin expression alternates with migrating neural crest cells in the trunk of the avian embryo. Development 111:15-22.

Ranscht B, Dours-Zimmermann MT (1991) T-cadherin, a novel cadherin cell adhesion molecule in the nervous system, lacks the conserved cytoplasmic region. Neuron 7:391-402.

Redies C, Inuzuka H, Takeichi M (1992) Restricted expression of Nand R-cadherin on neurites of the developing chicken CNS. J Neurosci 12:352.5-3534.

Redies C. Engelhart K, Takeichi M (1993) Differential expression of $\mathrm{N}$ - and R-cadherin in functional neuronal systems and other structures of developing chicken brain. J Comp Neurol 333:398-416.

Rickmann M, Fawcett JW, Keynes RJ (1985) The migration of neural crest cells and the growth of motor axons through the rostral half of the chick somite. J Embryol Exp Morphol 90:437-455.

Rogers SL, Letourneau PC, Palm SL, McCarthy J, Furcht LT (1983) Neurite extension by peripheral and central nervous system neurons in response to substrate-bound fibronectin and laminin. Dev Biol 98: 212-220.

Rogers SL, Edson KJ, Letourneau PC, McLoon SC (1986) Distribution of laminin in the developing peripheral nervous system of the chick. Dev Biol 113:429-435.

Rutishauser U, Grumet M, Edelman GM (1983) Neural cell adhesion molecule mediates initial interactions between spinal cord neurons and muscle cells in culture. J Cell Biol 97:145-152.

Sacristán MP, Vestal DJ, Dours-Zimmermann MT, Ranscht B (1993) T-cadherin 2: molecular characterization, function in cell adhesion, and coexpression with $\mathrm{T}$-cadherin and $\mathrm{N}$-cadherin. J Neurosci Res 34:664-680.

Sanes JR, Marshall LM, McMahon UJ (1978) Reinnervation of muscle fiber basal lamina after removal of myofibers. Differentiation of regenerating axons at original synaptic sites. J Cell Biol 78:176-198.

Silver J, Rutishauser U (1984) Guidance of optic axons in vivo by preformed adhesive pathways on neuroepithelial endfeet. Dev Biol 106:485-499.

Snow DM, Lemmon V, Carrino DA, Caplan AI, Silver J (1990) Sulfated proteoglycans in astroglial barriers inhibit neurite outgrowth in vitro. Exp Neurol 109:111-130.

Stern CD, Sisodiya SM, Keynes RJ (1986) Interactions between neurites and somite cells: inhibition and stimulation of nerve growth in the chick embryo. J Embryol Exp Morphol 91:209-226.

Stern CD, Norris WE, Bronner-Fraser M, Carlson GJ, Faissner A, Keynes RJ, Shachner M (1989) J1/tenascin-related molecules are not responsible for the segmented pattern of neural crest cells or motor axons in the chick embryo. Development 107:309-319.

Tanaka H, Agata A, Obata K (1989) A new membrane antigen revealed by monoclonal antibodies is associated with motor neuron axonal pathways. Dev Biol 132:419-435.

Tang J, Landmesser L, Rutishauser U (1992) Polysialic acid influences specific pathfinding by avian motor neurons. Neuron 8:1031-1044.

Tessier-Lavigne M, Mudge AW (1987) Distribution of calcitonin generelated peptide in chick motor neuron pools. Soc Neurosci Abstr 13: 1609.

Tosney KW (1988) Proximal tissues and patterned neurite outgrowth at the lumbosacral level of the chick embryo: partial and complete deletion of the somite. Dev Biol 127:266-286.

Tosney KW, Landmesser LT (1984) Pattern and specificity of axonal outgrowth following varying degrees of chick limb bud ablation. J Neurosci 4:2518-2527.

Tosney KW, Landmesser L (1985a) Growth cone morphology and trajectory in the lumbosacral region of the chick embryo. J Neurosci 5:2345-2358.

Tosney KW, Landmesser L (1985b) Development of the major pathways for neurite outgrowth in the chick hindlimb. Dev Biol 109:193214.

Tosney KW, Oakley RA (1990) The perinotochordal mesenchyme acts as a barrier to axon advance in the chick embryo: implications for a general mechanism of axonal guidance. Exp Neurol 109:75-89.

Tosney KW, Watanabe M, Landmesser L, Rutishauser U. (1986) The distribution of NCAM in the chick hindlimb during axon outgrowth and synaptogenesis. Dev Biol 114:437-452.

Vestal DJ, Ranscht B (1992) Glycosyl phosphatidylinositol-anchored $\mathrm{T}$-cadherin mediates calcium-dependent homophilic cell adhesion. $\mathrm{J}$ Cell Biol 119:451-461.

Volk T, Geiger B (1984) A 135-kd membrane protein of intercellular adherens junctions. EMBO J 3:2249-2260. 